\title{
UM ESTUDO INTER-COMPARATIVO DE PREVISÃO SAZONAL ESTATÍSTICA- DINÂMICA DE PRECIPITAÇÃO NO NORDESTE DO BRASIL
}

\author{
JOSÉ MARIA BRABO ALVES ${ }^{1,2}$, ALEXANDRE ARAÚJO COSTA ${ }^{1,3}$, SÉRGIO SOUSA SOMBRA ${ }^{1}$, \\ JOSÉ NILSON BEZERRA CAMPOS ${ }^{1,2}$, FRANCISCO DE ASSIS DE SOUZA FILHO ${ }^{1}$, \\ EDUARDO SÁVIO PASSOS RODRIGUES MARTINS ${ }^{1}$, EMERSON MARIANO DA SILVA ${ }^{3}$, \\ ANTÔNIO CARLOS SANTANA DOS SANTOS ${ }^{3}$, HUMBERTO A. BARBOSA ${ }^{1}$, \\ WAGNER LUIS BARBOSA MELCIADES ${ }^{1}$, DAVID FERRAN MONCUNNIL ${ }^{1}$ \\ ${ }^{1}$ Fundação Cearense de Meteorologia e Recursos Hídricos (FUNCEME) \\ Av. Rui Barbosa, 1246, Fortaleza-CE, CEP: 60.115-221 - brabo@funceme.br \\ ${ }^{2}$ Universidade Federal do Ceará (UFC), Centro de Tecnologia, \\ Departamento de Engenharia Hidráulica e Ambiental. \\ ${ }^{3}$ Universidade Estadual do Ceará (UECE), Departamento de Física, Fortaleza-CE
}

Recebido Abril 2006 - Aceito Junho 2006

\begin{abstract}
RESUMO
Apesar dos avanços da modelagem dinâmica da atmosfera nas últimas duas décadas do século 20 as aplicações com modelagem empírica ainda têm sido muito utilizadas em função do seu fácil uso e pouca dependência de recursos computacionais. Neste estudo é mostrada uma comparação dos resultados de simulação de precipitação para o Nordeste do Brasil (NEB) - 1971-2000 obtido por modelagem dinâmica de grande escala e regional (downscaling) e a previsão de um método de modelagem empírica K'neigbhors. Foram usados o modelo de circulação geral da atmosfera ECHAM4.5, e dois modelos regionais, o Modelo Regional Espectral (MRE/97) do National Centers for Atmospheric Prediction- NCEP e o Regional Atmospheric Model System (RAMS) desenvolvido na Universidade do Colorado. Os modelos regionais foram aninhados aos dados simulados pelo ECHAM4.5 tendo como condição de contorno a Temperatura da Superfície do Mar observada no período de fevereiro a maio. Os resultados mostraram que o modelo empírico apresentou menor erro absoluto nos períodos fevereiro a abril (FMA) e março a maio (MAM) que os modelos dinâmicos em áreas do no norte do Maranhão e Piauí, oeste e sul do Ceará, centro-sul do Piauí e oeste e nordeste da Bahia. Quanto ao bias, o ECHAM4.5 e o MRE/97 mostraram um bias úmido em grande parte do NEB, porém com um valor médio para o setor norte do NEB $\left(2^{\circ} \mathrm{S}-12^{\circ} \mathrm{S}\right.$ e $\left.45^{\circ} \mathrm{W}-37^{\circ} \mathrm{W}\right)$ mais próximo do observado. Para o RAMS e o método análogo houve um predomínio de um bias seco sobre o NEB, com valores em magnitudes mais distantes do observado. A previsibilidade (Heidke Skill) em três categorias Seca (S), Normal (N), Chuvosa (C), mostrou que o método análogo tem baixo Skill, entre 0,1 e 0,3 em todas as categorias, enquanto os modelos dinâmicos apresentaram Skill superiores, com valores maiores para as categorias $\mathrm{S}$ e C (da ordem de 0,4 a 0,5), até superiores a 0,6 em algumas áreas do setor norte do NEB para a categoria C como visto nos resultados dos modelos ECHAM4.5 e MRE/97.
\end{abstract}

Palavras-Chave: Análogos, Previsibilidade, Redução de escala.

ABSTRACT: A STUDY INTER-COMPARATIVE OF STATISTICAL-DYNAMICAL SEASONAL FORECAST PRECIPITATION IN NORDESTE'S BRAZIL.

Despite significant advances of the dynamic atmosphere models over the last decades of the $20^{\text {th }}$ century, the empirical atmospheric models have been widely used due mostly to both its general applicability and its little dependence on the computational resources. This study is show comparison of precipitation simulation to Northeast Brazil (NEB) - 1971-2000 from large scale dynamical modeling and regional model (downscaling) and the forecast of empirical modeling ( $K$-nearestneighbor (k-NN). Were user the general circulation model ECHAM4.5 together two regional models, the Regional Spectral Model (RSM/97) from the National Centers for Atmospheric Prediction-NCEP 
and the Regional Atmospheric Model System (RAMS) developed at Colorado State University. The regional models were nested in ECHAM4.5, forced with the observed Sea Surface Temperature as a boundary condition, for the period from February to May. The results show that the empirical model presented a smaller absolute error than the dynamic models for the periods February to April (FMA) and March to May (MAM) in isolated areas of the north of Maranhão and Piauí states, west and south of Ceará, center-south of Piauí and west and northeast of Bahia state. Regarding model biases, ECHAM4.5 and the RSM/97 produced, to a large extent, a humid bias over large areas of NEB, however with an average precipitation for the northern sector of NEB $\left(2^{\circ} \mathrm{S}-12^{\circ} \mathrm{S}\right.$ and $\left.45^{\circ} \mathrm{W}-37^{\circ} \mathrm{W}\right)$ close to the observations. RAMS and the analog method had a dominance of a dry bias over NEB, with precipitation totals below the observed values. The model skills (using the Heidke score) were evaluated for three categories Dry (S), Normal (N), Rainy (C), showed that the analogous method has low skills, between 0,1 and 0,3 in all categories, while dynamic models presented superior skills, with larger values for categories $\mathrm{S}$ and $\mathrm{C}$ (of the order of 0,4 the 0,5 ), exceeding 0,6 in some areas of the northern sector of NEB for category $\mathrm{C}$ as seen in the models ECHAM4.5 and RSM/97 models.

Keywords: Analogs, Previsibility, Downscaling.

\section{INTRODUÇÃO}

Técnicas que abordam a previsão de eventos climáticos evoluíram significativamente nesses últimos 20 anos. Revisões sobre o desempenho de previsões dinâmicas e empíricas podem ser encontradas em Hastenrath (1985), (1990), (1995), (1995a), (1995b), (1996), (2002), Palmer e Anderson (1994); Carson (1998), Latifi et al. (1998), Anderson et al. (1999) e Goddard et al. (2001). O desenvolvimento da modelagem dinâmica com o aperfeiçoamento dos modelos numéricos de circulação geral da atmosfera (MCGAs), e os modelos regionais de escala espacial limitada (MRs) têm contribuído para diagnosticar e prever eventos meteorológicos de tempo e clima em várias áreas do globo com melhor desempenho (Giorgi e Bates, 1989; Giorgi, 1990; Cavalcanti et al., 2002; Chou et al., 2002; Marengo et al., 2003, Nobre et al., 2001; Sun et al, 2005).

Apesar do avanço da modelagem dinâmica o seu custo financeiro ainda é relativamente alto, embora o aparato computacional tenha diminuído de preço ao longo dos anos. A modelagem numérica por MCGAs requer, geralmente, supercomputadores quase nem sempre disponíveis na maioria dos centros operacionais de meteorologia. Modelagem por MRs são menos dispendiosas, mas em geral necessitam de clusters de computadores pessoais (Pcs) e analistas/especialistas em modelagem numérica. Além disso, a modelagem dinâmica requer uma alimentação de dados em tempo real de variáveis atmosféricas e de superfície que em algumas situações não estão disponíveis.

Basicamente, são utilizados dois tipos de modelos na modelagem atmosférica. Os MCGAs, que são modelos globais (resolução espacial igual ou acima de 250 quilômetros) e os modelos regionais (MRs) que são modelos de área limitada, com resolução variável da ordem de dezenas de quilômetros.
Esses MRs, por serem de área limitada, também incorporam, ou podem incluir, características mais refinadas da região de interesse, como topografia, vegetação, solo, etc. Além da resolução de processos físicos, por exemplo, balanço de radiação em nuvens, microfísica de nuvens, etc., que nos MCGAs são parametrizados. No que se refere ao desempenho é esperado que os dos MRs sejam superiores do que os MCGAs. Nos últimos anos, uma nova técnica chamada de downscaling, que usa MRs aninhados a MCGAs, apresentou resultados promissores na configuração e intensidade de variáveis atmosféricas para escalas regionais, nas quais os MCGAs têm pouca sensibilidade (Giorgi e Bates, 1989; Nobre et al., 2001; Sun et al., 2005; Alves et al. 2003, 2005).

Uma alternativa a modelagem dinâmica para estudos diagnósticos e de previsão de variáveis meteorológicas, devido a sua facilidade de aplicação, tem sido o uso de técnicas estatísticoestocásticas. Essas técnicas tentam predizer o estado de uma ou mais variáveis atmosféricas tendo como bases relações entre essas variáveis que guardam algum sinal físico entre as mesmas.

Muitos estudos na literatura meteorológica têm mostrado que o uso das técnicas estatístico-estocásticas como métodos de prognósticos apresentaram resultados com um melhor desempenho do que os advindos da modelagem dinâmica. Nessa linha têm destaque os estudos de Barnston et al. (1994, 1999), Anderson et al. (1999) e Bamzai e Shukla (1999), Greischar e Hastenrath (2000).

Mais específico, para o Nordeste do Brasil (NEB), alguns estudos de modelagem estatístico-estocástica têm mostrado que esta tem melhor desempenho do que os resultados da modelagem dinâmica para a previsão de chuva sazonal nessa região. Folland et al. (2001), compararam vários resultados de previsão estatístico-estocástica de precipitação para o NEB, usando diferentes preditores para um período de quatro anos, 
com resultados de modelagem numérica e observaram que os seus resultados da modelagem não superaram o desempenho do modelo empírico.

Moura e Hasteranth (2004), apresentaram uma comparação entre a previsão de chuva (período de março a junho) para o NEB, resultante de uma modelagem empírica desenvolvida na Universidade de Wisconsin (Hastenrath e Greischar, 1993a, 1993b) e os resultados de um MCGA (ECHAM4.5) para um período de 1968-2002. Seus resultados, também, mostraram um melhor desempenho do modelo empírico em relação ao modelo numérico. Souza Filho e Lall (2003 e 2004) desenvolveram metodologia semi-paramétrica para a previsão de vazões aplicando a técnica dos K' vizinhos (K'neighbors). Estes autores observaram que havia previsão nestes artigos a partir de Julho, isto é, seis (6) meses antes da estação chuvosa.

Os estudos que utilizam aproximações por K'neighbors têm sido muito usados em simulação e previsão de variáveis atmosféricas e hidrológicas pela facilidade de sua aplicação. Essa técnica basicamente simula ou prevêem variáveis com base na relação com outras variáveis e com a sua própria característica observada em anos passados (Souza Filho e Lall, 2003; Lall e Sharma, 1996). Young (1994) apresentou um estudo de re-amostragem de simulações de séries diárias de temperatura máximas e mínimas e precipitação usando a técnica de K'neighbors. Lall e Sharma (1996) discutiram uma aproximação por $K^{\prime}$ neighbors para gerar séries hidrológicas temporais. Rajagopalan e Lall (1999) usaram o algoritmo de Lall e Sharma (1996) para geração de precipitação diária e outras cinco variáveis atmosféricas. A técnica de $K^{\prime}$ neighbors mostrouse capaz de reproduzir várias estatísticas simples e a distribuição de dias secos e chuvosos da precipitação. Uma simulação diária para vários locais, na bacia do Rio Reno, usando K'neighbors, para precipitação e temperatura foi discutida em Buishand e Brandsma (2001).

O objetivo desse estudo é comparar a previsão de chuva trimestral fevereiro-março-abril (FMA), março-abril-maio (MAM) e fevereiro-março-abril-maio (FMAM), obtida de um método de modelagem empírica ( $K^{\prime}$ neigbhors), com a de modelos dinâmicos (um MCGA - ECHAM4.5, e de MRs aninhados (downscaling) ao ECHAM4.5, o Modelo Regional Espectral (MRE/97) e RAMS (Regional Atmospheric Model System).

\section{DADOS E METODOLOGIA}

\subsection{Dados de Precipitação}

Dados contínuos de precipitação para grandes períodos de anos, com um extenso número de estações, com boa qualidade e com elevada cobertura espacial ainda são escassos sobre o NEB e Atlântico Tropical (Silva Filho, 2005). Os dados usados nesse estudo para servirem como fonte observacional e de comparação com os resultados da modelagem estatístico-estocástica e dinâmica tiveram como base uma composição de vários conjuntos observacionais. O objetivo foi tentar diminuir os problemas mencionados acima. A base foi o arquivo disponível na Universidade de East Anglia (UEA) no reino Unido (New et al., 2000). Estes dados foram compilados com o uso de mais de 19.800 estações de observações ao longo do globo e interpolados para uma grade global de $0,5^{\circ}$ de latitude e longitude, com um acurado controle de qualidade e estão disponíveis como totais mensais desde 1901-1996, mas somente o período de 1971-1996 foi usado nesse estudo, sendo os mesmos interpolados para a grade dos modelos ECHAM4.5, MRE/97 e RAMS.

Adicionalmente a esse conjunto de dados da $U E A$ foram usados totais de precipitação sobre o Brasil do Instituto Nacional de Meteorologia (INMET) interpolada para uma grade $0,25^{\circ}$ de latitude e de longitude, para um período de 1994-2000. Estes dados do INMET foram também interpolados para a mesma grade dos modelos acima mencionados. Algumas estações de observações que serviram de base para formação desse arquivo de dados do INMET são diferentes das do conjunto de dados da UEA, e a densidade de estações usadas também foi maior do que da $U E A$. Entretanto com um controle de qualidade inferior (Sun et al., 2005). Nos pontos de grade onde havia dados dos dois conjuntos esses receberam pesos iguais na sua utilização. Após este procedimento este conjunto de dados foi novamente interpolado espacialmente (pelo método do inverso do quadrado da distância) para a grade do modelo RAMS (aproximadamente com uma resolução de $1^{\circ} \times 1^{\circ}$ de latitude e longitude sobre a região do $\mathrm{NEB}$ ).

\subsection{Técnica Estatístico-Estocástica Usada no Estudo}

Foi usado um método estatístico-estocástico que tem como base a relação entre essa precipitação no NEB e variáveis climáticas que são observadas nos Oceano Pacífico e Atlântico Tropical (Hastenrath e Heller, 1977; Moura e Shukla, 1981, Nobre e Shukla, 1996).

O método utilizado, com o uso das informações de preditores climáticos, foi similar aos usados por Souza Filho e Lall (2003) e Cardoso (2003). Os chamados preditores climáticos, em geral, são previamente conhecidos (Moura e Shukla, 1981) sendo atribuídos pela relação entre suas variabilidades e a precipitação ao longo de anos.

Após as análises de correlações, em função dos preditores e precipitação no NEB, foram escolhidas áreas que apresentaram as maiores correlações, essas foram usadas na identificação dos análogos. As regiões selecionadas foram: Niño 3 (definida como a anomalia de temperatura média mensal da superfície do mar na região do Pacífico Equatorial entre $150^{\circ} \mathrm{W}$ e $90^{\circ} \mathrm{W}$ e $5^{\circ} \mathrm{S}$ e $5^{\circ} \mathrm{N}$ ) e um gradiente de temperatura da superfície do mar (TSM) do Atlântico Tropical (GAT) definida como a 
diferença na média mensal da anomalia de TSM na região do Atlântico Norte $\left(5-20^{\circ} \mathrm{N}, 60-30^{\circ} \mathrm{W}\right)$ e a média mensal da região do Atlântico $\mathrm{Sul}\left(0-20^{\circ} \mathrm{S}, 30^{\circ} \mathrm{W}-10^{\circ} \mathrm{E}\right)$. Essas áreas coincidem com as que têm maior impacto nas chuvas do NEB (Moura e Shukla, 1981; Uvo et al; 1998). A este gradiente de TSM chamase também de dipolo de TSM do Atlântico Tropical. Quando o GAT é positivo apontando para o Atlântico Norte denomina-se o mesmo como dipolo positivo de TSM, contrário quando esse é negativo na direção sul chama-se o mesmo de dipolo negativo de TSM (Moura e Shukla., 1981). Os dados das anomalias de TSM nas áreas de Niño3 e do dipolo de TSM estão disponíveis no site do Climate Prediction Center.

A base metodológica dos chamados métodos dos vizinhos (K-neighbors) é explicada a seguir. A identificação do análogo tem como base o valor da distância Euclidiana (di) entre $\mathrm{o}$ valor do vetor dos preditores atuais $\mathrm{x}^{*} \mathrm{e} \mathrm{o}$ valor dos preditores em sua série histórica $\mathrm{x}_{\mathrm{i}}$, na seguinte forma:

$$
\mathrm{d}_{\mathrm{i}}{ }^{2}=\sum_{\mathrm{j}=1}^{\mathrm{j}=2}\left\{\left(\mathrm{x}_{\mathrm{j}}{ }_{\mathrm{j}}-\mathrm{x}_{\mathrm{i}, \mathrm{j}}\right)\right)^{2},
$$

Onde: $\mathrm{x}^{*}$ é um vetor $1 \mathrm{x} 2, \mathrm{x}_{\mathrm{i}}$ é um vetor $1 \mathrm{x} 2$ de preditores durante o i-ésimo ano usado. As distâncias assinalam a similaridade da condição de preditor atual com cada uma das condições passadas. Segundo Souza Filho e Lall (2003), com aplicação posterior de Cardoso (2003) para a previsão de vazão e operação de reservatórios hídricos no estado do Ceará, o vetor distância d determina o conjunto de valores mais próximos aos preditores atuais $\mathbf{x}^{*}$ (correspondente ano de previsão), identificando a posição em um ordenamento dos vizinhos mais próximos (o elemento j está associado ao j-ésimo $\mathbf{x}$ mais próximo de $\mathbf{x}^{*}$ ).

A partir desse passo selecionam-se o número de vizinhos $K$ 's a serem utilizados e suas respectivas funções pesos (ou probabilidades) $\mathrm{K}(\mathrm{j})$ a ser associada a cada um dos $\mathrm{K}$ vizinhos. Abaixo são mostradas algumas funções pesos que podem ser utilizadas.

$$
\begin{aligned}
& \mathrm{K}_{(\mathrm{j})}=1 / \mathrm{k} \quad \text { (uniforme), } \\
& \mathrm{K}_{(\mathrm{j})}=\frac{1 / \mathrm{j}}{\sum_{\mathrm{i}=1}^{\mathrm{k}} \frac{1}{\mathrm{i}}},(\text { Lall e Sharma, 1996), }
\end{aligned}
$$

Por exemplo, a formulação de Lall e Sharma (1996) calcula o peso pela posição em relação ao ano de previsão, isto é o peso do ano mais próximo é $K(1)=(1 / 1) /(1 / 1+1 / 2+\ldots \ldots \ldots$. $1 /$ nk). A função de peso $K(j)=1 / k$ distribui os pesos de forma uniforme, ou seja $\mathrm{k}(1)=1 / \mathrm{nk}$. Esta última equação considera a distância Euclidiana entre o ano de previsão e cada um dos anos próximos. É importante mencionar que nosso estudo, os $K^{\prime} s$ foram identificados no mês de novembro, dois meses antes do início da estação chuvosa do norte do NEB (região de interesse para a previsão de chuva). Após a identificação dos
$K^{\prime} s$, para cada ano entre $1971-2000$, os $10 K^{\prime} s$ mais próximos das anomalias observadas em novembro para a região de Niño3 e para o dipolo de TSM no Atlântico Tropical foram usados como preditores. Nesse caso, como o análogo usado foi relativo a novembro e a previsão de chuva é fevereiro a maio do ano seguinte, os somatórios dos trimestres FMA e MAM e do quadrimestre fevereiro, março, abril e maio (FMAM) do ano seguinte aos 10 análogos multiplicados pelas suas respectivas funções pesos (fórmulas 2 e 3), foi o valor do trimestre (quadrimestre) prognosticado para o ano em questão entre 1971-2000. Os testes feitos nesse estudo mostraram que a função peso (2) obteve melhores resultados em relação ao observado, sendo a mesma usada para a previsão.

\subsection{Sobre o MCGA e os Modelos Regionais Usados no Estudo}

\subsubsection{MCGA $E C H A M 4.5$}

Segundo Sun et al. (2005) o AGCM usado neste estudo é o European Comunity-Hamburg (ECHAM) versão 4.5. desenvolvido no Max Planck Institute for Meteorology and German Climate Computing Centre (DKRZ). Esse modelo é baseado no modelo de previsão de tempo do European Centre for Medium Range Weather Forecasts (ECMWF). Várias modificações têm sido aplicadas no $D K R Z$ para melhorar a previsão climática, estando o mesmo atualmente na quarta geração. Uma detalhada descrição do ECHAM4.5 pode ser encontrada em Roeckner (1996). O ECHAM4.5 é um dos modelos usados operacionalmente na previsão climática sazonal no International Research Institute (IRI), e o mesmo tem tido bom desempenho na simulação da variabilidade climática sobre o nordeste da América do Sul (http://iri.columbia.edu/forecast/skill/SkillMap.html).

$\mathrm{Na}$ versão padrão do modelo o mesmo tem 19 níveis híbridos usados em um sistema de coordenadas de pressãosigma. O domínio vertical estende-se até o nível de pressão de $10 \mathrm{hPa}$. Variáveis prognósticas são vorticidade, divergência, logaritmo de pressão a superfície, temperatura, umidade específica, razão de mistura e total de água de nuvem. Exceto para componentes de vapor o prognóstico das variáveis são representados por harmônicos esféricos com truncamento triangular no número de onda T42.

O passo de tempo para a dinâmica e a física é de 24 minutos para a resolução horizontal T42. O passo de tempo para a radiação são duas horas. Os ciclos diurno e sazonal da forçante solar são simulados. Para o transporte de vapor d'água e água de nuvem um semi-lagrangeano esquema é usado. Os fluxos turbulentos de superfície são calculados através do Monin-Obukov bulk usando teoria da similaridade (Louis, 1981). Dentro e acima da camada limite atmosférica um esquema fechado de alta ordem é usado para computar a transferência turbulenta 
de momento, calor, mistura e água de nuvem. Os coeficientes de difusão turbulenta são calculados como função da energia cinética turbulenta (E). O modelo de solo contempla os balanços de calor, água no solo, pacote de neve sobre o continente e balanço de calor de gelo sobre o continente.

Efeitos de vegetação tais como interceptações de chuva na copa das árvores e controles de evapotranspiração pelos estomatos são parametrizados. O esquema de estimativa do escoamento superficial tem como base a área de captação da bacia hidrográfica e leva em consideração as variações de sub-grade da capacidade máxima de armazenamento de água no solo (capacidade de campo) sobre áreas continentais não homogêneas.

Parâmetros de superfície tais como albedo, rugosidade, tipo de vegetação, índice de área foliar e parâmetros de solo tais como, capacidade de armazenamento de água e condutividade térmica tem suas compilações segundo Claussen et al., 1994. As parametrizações de convecção cumulus (rasa, média e profunda) são baseadas no conceito de fluxo de Tiedtke (1989). Um conjunto de 10 integrações do ECHAM4.5 forçadas com TMSs observadas foram executadas no IRI desde o início dos anos 40 até o presente.

\subsection{2 - Modelo Regional Espectral (MRE/97)}

O MRE aqui usado é uma versão do MR de 1997 desenvolvidos no Centro de Modelagem de Meio Ambiente do NCEP desenvolvidos por Juang e Kanamitsu (1994). Uma importante característica do MRE é a resolução da perturbação dependente do tempo e o desempenho de alta ordem da computação que utiliza o método espectral. O MRE tem a mesma física do modelo espectral global (MEG) que é usado para previsão de médio prazo como descrito em Kanamitsu et al. (1991).

Um importante avanço foi também endereçado na dinâmica do MR para manter a sua estabilidade computacional, uma opção de difusão local contida no MEG foi implementada no mesmo (Iridell e Purser, 1994). A mesma pode ser utilizada para passos de tempo mais longos e faz uma checagem e difusão das áreas de ventos fortes que poderiam causar instabilidade computacional. Essa opção pode não ser ideal para previsão em áreas onde correntes de jato são importantes dinamicamente para sistemas atmosféricos de curta duração, entretanto pode ser útil para estudos climáticos que requerem integrações mensais com MR de alta resolução.

O MRE tem 18 níveis sigma na vertical, com as seguintes características físicas: radiação de onda longa e onda curta com variação diurna, interação com radiação de nuvem, uma camada na superfície com propriedades físicas da camada limite planetária, arrasto por onda de gravidade, convecção cumulus tipo Arakawa-Shubert simplificada (Pan e Wu, 1994; Hong e Pan, 1996), convecção rasa e alguns processos hidrológicos (Kanamitsu, 1989).
As parametrizações físicas são computadas a cada passo de tempo, exceto para a rotina de radiação que é chamada a cada 1 hora pelo MRE. Além disso, têm sido testado e implementado pacotes físicos, particularmente, para parametrização de convecção (Juang et al., 1997). O MRE usa um modelo de solo de duas camadas descrito por Pan e Marth (1987). Esse modelo de solo é usado para descrever o papel da vegetação e sua interação com a umidade no solo em modificar as trocas entre a superfície-atmosfera de fluxos de momento, energia e vapor d'água.

\subsubsection{Regional Modeling Atmospheric System (RAMS)}

O Sistema de Modelagem Atmosférica Regional, RAMS (Regional Atmospheric Modeling System) foi desenvolvido pelo Departamento de Ciências Atmosféricas da Universidade Estadual do Colorado, USA. O modelo RAMS é muito flexível e com muitas opções, podendo ser configurado para fazer simulações da circulação atmosférica em várias escalas de tempo e espaço, bem como diferentes resoluções horizontal e vertical. O modelo RAMS é do tipo euleriano (Pielke et al., 1992 e Tramback, 1990).

A micro-física do RAMS é de volume, explícita, com esquema de um ou dois momentos. No primeiro esquema (usado neste estudo), a razão de mistura é prognosticada e a concentração ou tamanhos dos hidrometeoros são especificados. No segundo caso, tanto a razão de mistura como a concentração como o tamanho dos hidrometeoros é prognosticado. O pacote de microfísica contém sete classes de hidrometeoros (água de nuvem, água de chuva, cristais de gelo, neve, agregados, graupel e granizo, Walko et al., 1995). Um esquema radiativo acoplado a microfísica que compreende três bandas de radiação de onda curta e cinco de onda longa foi usado (Harrington, 1997; Olson et al., 1998). O esquema de superfície foi o LEAF-2, no qual diferentes tipos de vegetação podem ser introduzidos em cada coluna do modelo para representar a escala de sub-grade. O esquema de turbulência usado foi o de Mellor e Yamada (1975), devido à grade do estudo ser de dezenas de quilômetros (seção 2.4).

O modelo é escrito em coordenado vertical sigma, que segue o contorno da topografia. Maiores detalhes da física do Modelo RAMS são encontrados em Pielke et al. (1992) e Tremback (1990). O modelo emprega os esquemas Leapfrog, avançado-recuado ou a combinação híbrida na resolução de suas equações. Maiores detalhes de todos os processos físicos envolvidos nesse sistema de modelagem e suas opções de uso podem ser encontrados em Chen e Cotton (1983, 1987), Harrington (1997); Walko et al., (1995), Walko (2000) e outros. Segundo Costa (2006) o modelo RAMS foi usado com sucesso em simulações de convecção tropical e de latitudes médias (Alexander e Cotton, 1998; Ziegler et al., 1997; Olsson e Cotton, 1997; Nair et al., 1997 e Costa et al. 2001 a,b). 


\subsection{Sobre os Experimentos de Modelagem Numérica de Grande Escala e Regional}

Vale mencionar que os resultados desse estudo se deterão a comparar resultados de modelagem dinâmica no chamado modo de simulação. Esse modo na literatura meteorológica é entendido como o modo de "previsão" perfeita, visto que se usa a TSM observada com condição de contorno a superfície para os modelos numéricos.

Segundo Alves et al. (2005), o aninhamento entre o MCGA ECHAM4.5 e o MRE/97 foi unidirecional usando os resultados do MCGA de janeiro a maio (1971-2000), como dados de entrada para o MRE/97 de 6 em 6 horas. O método de perturbação aninhada usada seguiu o utilizado no MCGA sobre todo o domínio, e não somente na zona de fronteira lateral.

Essa metodologia é diferente dos métodos convencionais usados na modelagem numérica, que usam os resultados do MCGA somente nas fronteiras laterais dos MRs. As variáveis dependentes no MRE são definidas como a soma da perturbação e o campo base (campo que depende do ajuste do MCGA em relação ao MRE). Maiores detalhes sobre o MRE/97 podem ser encontrados em Juang et al. (1997).

Antes da execução do downscaling (1971-2000) foram executadas algumas rodadas testes para o mês de abril de 2001 . Essas serviram para a escolha do domínio regional a ser utilizado, que compreende o comprimento ideal para fins de recursos computacionais, além da área geográfica específica de localização da fronteira lateral. Essas áreas compreendem áreas de topografia plana e sem grande atividade convectiva (formação de nuvens profundas), características que afetam a sensibilidade dos MRs. Após uma análise comparativa do campo de precipitação sobre o NEB e bacia do Atlântico Tropical entre o observado e o simulado, optou-se pela escolha da rodada de número 7 (Tabela 1) para ser usada como controle para execução do downscaling do MRE/97 para os meses de janeiro a maio de 1971. Detalhes dessa rodada teste podem ser vistos na Tabela 1.

Essa simulação foi que apresentou melhor configuração qualitativa e um menor bias no campo de chuva sobre o NEB e bacia do Atlântico Tropical. Os domínios do MCGA e do MR/97 usados na simulação de número 7 e suas topografias utilizadas são mostrados nas Figuras 1 e 2.

O MRE/97 foi executado, com um conjunto de 10 membros, na versão não-hidrostática, passo de tempo 600 segundos em modo de simulação. O espaçamento de grade foi de $60 \mathrm{~km}$, centrado na latitude de $3^{\circ} \mathrm{S}$ e na longitude de $27^{\circ} \mathrm{E}$ e 18 níveis verticais, e o domínio compreendeu desde o oeste da África do Sul até Região Amazônica (Figura 1), para um período seis meses (janeiro a junho) durante 30 anos, de 1971 a 2000. Nas simulações o tipo de solo foi um com característica intermediária entre um arenoso e um argiloso. A vegetação foi do tipo savana com uma cobertura de $70 \%$ da área de cada grade do MRE. Para a cobertura do tipo de solo essa foi similar a usada para vegetação. A topografia usada pelo MRE/97, comparada com aquela na resolução do modelo global é mostrada na Figura 2 para a região do NEB e vizinhanças.

A grade do RAMS escolhida foi uma polar estereográfica de 80 pontos nas direções horizontais (latitudinais e longitudinais) com espaçamento de $40 \mathrm{~km}$. Esta grade foi centrada em $10^{\circ} \mathrm{S}$ e $40^{\circ} \mathrm{W}$ possibilitando o incluir todo o NEB e parte do Atlântico adjacente. Na vertical, de acordo com Costa (2006), para que fosse proporcionada uma boa representação do transporte convectivo, bem como de troca dos processos físicos na camada limite, usou-se uma grade vertical com quarenta e um níveis, com espaçamento variável entre $80 \mathrm{~m}$ (próximo à superfície) e $1 \mathrm{~km}$ em altos níveis da atmosfera. A grade vertical do modelo de solo continha 11 níveis.

O RAMS foi inicializado em modo de simulação com os dados do ECHAM4.5, sendo esta feita com um esquema de relaxamento Newtoniano (nudging), através de um termo adicionado as equações básicas, que faz com que o valor de cada variável nos diversos pontos de grade tenda àquele contido na informação de grande escala. A assimilação pode ser feita pelas fronteiras laterais do modelo (nudging lateral), ou em todo o domínio do modelo (nudging central). O contorno topográfico usado pelo RAMS é similar ao do MRE/97, mostrado na Figura 2 , mas numa resolução mais fina.

Tabela 1 - Experimentos de simulação executados para o mês de abril de 2001 para se definir o experimento de controle que serviu de base para o downscaling do MRE para o quadrimestre (fevereiro a maio - 1971 a 2000).

\begin{tabular}{lcccccccc}
\hline Rodadas & $\mathbf{1}$ & $\mathbf{2}$ & $\mathbf{3}$ & $\mathbf{4}$ & $\mathbf{5}$ & $\mathbf{6}$ & $\mathbf{7}$ & $\mathbf{8}$ \\
\hline Esp. Grade (km) & 30 & 40 & 60 & 80 & 40 & 60 & $\mathbf{6 0}$ & 60 \\
Passo de tempo (s) & 240 & 300 & 300 & 360 & 300 & 300 & $\mathbf{6 0 0}$ & 300 \\
Centro da grade & $3 \mathrm{~S}, 40 \mathrm{~W}$ & $3 \mathrm{~S}, 40 \mathrm{~W}$ & $3 \mathrm{~S}, 40 \mathrm{~W}$ & $3 \mathrm{~S}, 40 \mathrm{~W}$ & $3 \mathrm{~S}, 40 \mathrm{~W}$ & $3 \mathrm{~S}, 58 \mathrm{~W}$ & $\mathbf{3 S}, \mathbf{2 7} \mathrm{W}$ & $3 \mathrm{~S}, 40 \mathrm{~W}$ \\
No. de pontos X & 73 & 55 & 73 & 55 & 73 & 109 & $\mathbf{1 0 9}$ & 109 \\
No. de pontos Y & 90 & 68 & 72 & 54 & 90 & 72 & $\mathbf{7 2}$ & 72 \\
Duração (hora) & $16: 00 \mathrm{~h}$ & $06: 35 \mathrm{~h}$ & $10: 00 \mathrm{~h}$ & $04: 10 \mathrm{~h}$ & $13: 00 \mathrm{~h}$ & $17: 10 \mathrm{~h}$ & $\mathbf{1 7 : 1 0 h}$ & $17: 10 \mathrm{~h}$ \\
\hline
\end{tabular}


Antes da rodada de simulação dos 30 anos (1971-2000) para os 10 membros do ECHAM4.5, foi feito um teste usando apenas um membro para os anos de 1983 (muito seco) e 1985 (muito chuvoso) sobre o NEB para os meses de janeiro a junho. Esses testes envolveram microfísca de nuvens (completa versus somente fase quente, completa versus ausência de microfísica que consiste em somente convecção cumulus), esquemas de radiação (Harrington versus Chen-Cotton e versus MaherPielke), e teste com nudging central desligado e ligado.

De acordo com os resultados para ambos os anos com algumas simulações em que diferentes esquemas de microfísica e radiação e/ou radiação foram testados, o modelo não permaneceu estável e não produziu resultados de precipitação realistas. A combinação de uma microfísica completa (incluindo a fase de gelo) e com o esquema de radiação de Harrington (acoplado a microfísica) teve melhores resultados e foi usada nas simulações dos 30 anos (1971-2000). No caso do teste do nudging central o modelo mostrou resultados coerentes, tanto quando o mesmo esteve ligado quanto este foi desligado reproduzindo as diferenças de precipitação no NEB nos anos de 1983 e 1985, apesar da precipitação ser bem mais real no caso em que o nudging central foi ligado sendo esse adotado no estudo.

As forçantes de grande escala utilizadas nas simulações do MRE/97 e RAMS foram obtidas do conjunto das 10 integrações do MCGA ECHAM4.5 feitas no IRI para os meses de janeiro a junho de 1971 a 2000. As informações de larga escala das componentes meridional e zonal, umidade específica, temperatura e pressão a superfície serviram de entrada a cada 6 horas para alimentar o domínio do MRE/97. Temperatura da Superfície do Mar (TSM) observada nos oceanos Pacífico, Atlântico e Índico nos meses de janeiro a junho (1971-2000) serviram de variáveis de contorno à superfície nas simulações do ECHAM4.5, MRE/97 e do RAMS.

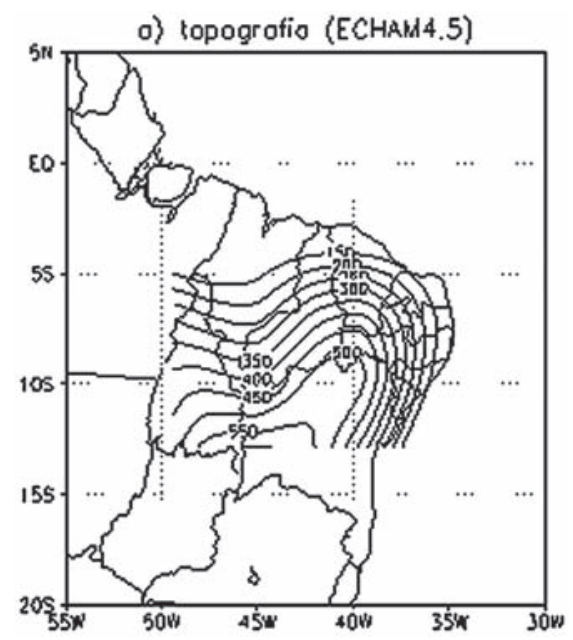

Figura 1 - Domínio dos modelos MCGA (ECHAM4.5) utilizado nas simulações do ECHAM4.5 e do MRE/97 (área A1 da figura).

Fonte: Alves et al., 2003.
Segundo Costa (2006), depois de escolhidos os esquemas numéricos e parametrizações físicas mais adequadas e constando-se que o RAMS apresentava sensibilidade significativa com respeito ao nudging central, foram realizadas simulações de climatologia (30 anos) com o uso de um membro do ECHAM4.5. Essas simulações objetivaram testar três configurações: sem nudging central, com nudging central fraco (assimilação em uma escala de tempo de 6 horas), e com nudging central forte (assimilação de 3 horas). De acordo com os resultados do Heidke Skill para três e cinco categorias de percentis de precipitação no NEB e os valores absolutos de precipitação mais reais, a configuração com nudging central fraco teve melhor desempenho sendo essa usada nas simulações dos 10 membros para os 30 anos (1971-2000).

\subsection{Parâmetros de Avaliação de Desempenho dos Modelos}

Três critérios foram usados para medir o desempenho da modelagem dinâmica e do método análogo no período de 1971-2000 para cada trimestre (FMA e MAM) para a estação chuvosa período de fevereiro a maio (FMAM). O bias, que é uma medida do erro sistemático do método, dado pela formulação abaixo.

$$
\mathrm{B}_{\mathrm{i}, \mathrm{j}}=\frac{1}{\mathrm{n}}\left(\mathrm{P}_{\mathrm{i}, \mathrm{j}}-\mathrm{O}_{\mathrm{i}, \mathrm{j}}\right) \text {, onde }
$$

$n$ é o número de anos de avaliação, os índices $i$ e $j$, indicam as coordenadas dos pontos de grade, e $P$ e $\mathrm{O}$ são os valores previstos e o observado.

O segundo parâmetro foi o erro médio absoluto (EMA) dado pela equação 5. Segundo Sun et al. (2005) o EMA é menos sensível que o erro médio quadrático a grandes desvios que se afastam da média. De acordo com Hansen et al. (2004). O EMA é considerado a mais robusta medida de desempenho.

$$
\mathrm{EMA}_{\mathrm{i}, \mathrm{j}}=\frac{1}{\mathrm{n}} \sum_{\mathrm{i}=1}^{\mathrm{n}}\left|\mathrm{P}_{\mathrm{i}, \mathrm{j}}-\mathrm{O}_{\mathrm{i}, \mathrm{j}}\right| \text {, onde }
$$

$n$ é o número de anos de avaliação, e $i$ e $j$ é o valor da variável em cada grade do domínio, e $P$ e $\mathrm{O}$ são os valores previstos e o observado. Um mapa síntese, em função do menor EMA foi elaborado. Nesse mapa é feita uma comparação do valor do EMA de cada modelo e do método análogo, considerando para o referido ponto de grade do domínio sobre o NEB. Uma cor de referência foi associada ao menor valor do EMA, e ao seu respectivo modelo ou método. Essa análise serviu para identificar ao longo do NEB que método teve menor diferença quantitativa entre a precipitação observada e a prognosticada no período de 1971-2000. Foram calculados também para uma área representativa do setor norte do NEB $\left(2^{\circ} \mathrm{S}-12^{\circ} \mathrm{S}\right.$ e $45^{\circ} \mathrm{W}-$ $37^{\circ} \mathrm{W}$ ) a média, o desvio padrão e o bias. 

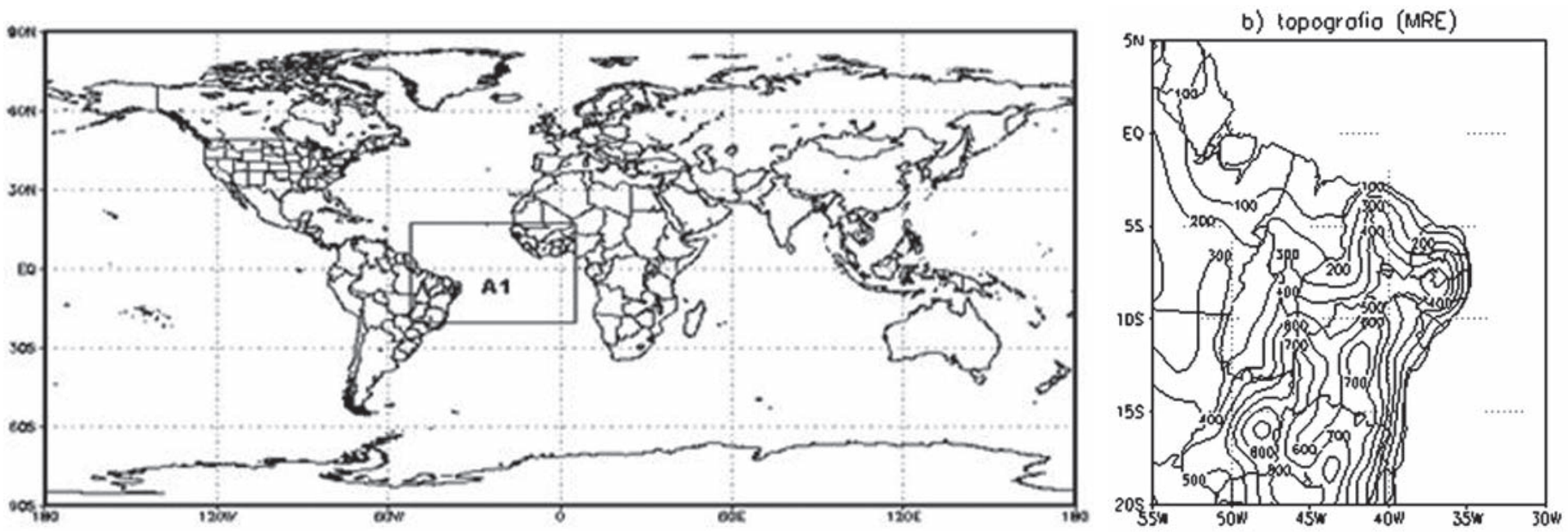

Figura 2 - Topografia sobre a Região Nordeste (m). (a) MCGA (ECHAM4.5), (b) MRE. O espaçamento entre as isolinhas da Figura 2a é de 50 m, e da 2 b é de 100 m. Fonte: Alves et al., 2003.

O desempenho da previsibilidade para três categorias de percentis foi medida pelo Heidke Skill (Wilks, 1995). O intervalo em (mm) de precipitação que definiu os limites de cada categoria para os dados simulados e observados no período (1971-2000) em cada ponto de grande do domínio sobre o NEB foi definido segundo a técnica dos percentis calculados por Xavier e Xavier (1998). Os limites segundo a aplicação de uma distribuição Gama são de $<=35 \%$ (Seco-S), $>35 \%$ a $<=65 \%$ (Normal-N), $>65 \%$ (Chuvoso-C). A formulação do Heidke Skill calculado em cada ponto de grade é mostrada abaixo.

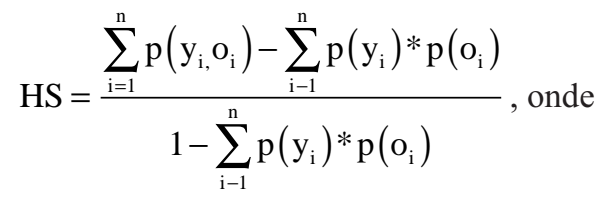

$y$ e o representa as previsões, $o$ as observações, $i$ o ano de comparação e $n$ o número de anos usados na comparação. Por exemplo, para a categoria Normal, $P\left(y_{i}, O_{j}\right)$ é a freqüência relativa de acertos entre o previsto e observado, isto é foi previsto Normal e observado foi Normal, $p\left(y_{i}\right)$ indica o somatório das freqüências relativas em que foi previsto Normal e observou-se Seco ou Chuvoso, e $p\left(o_{i}\right)$ indica o somatório das freqüências relativas em que foi previsto Seco ou Chuvoso e o observado foi Normal.

\section{RESULTADOS E DISCUSSÃO}

Todos os resultados apresentados foram interpolados para a grade do modelo RAMS. As figuras 3 e 4 mostram o bias do ECHAM4.5, do RSM/97, do RAMS e do método análogo para os trimestres FMA e MAM. Para o trimestre FMA notase que o ECHAM4.5 tem um bias úmido em grande parte do NEB predominando valores entre 0 e $2 \mathrm{~mm} /$ dia em direção a Amazônia e interior do NEB, e no oeste dos estados da Paraíba e Pernambuco o bias é seco com valores entre 0 e $-2 \mathrm{~mm} /$ dia (Figura 3a).

O MRE/97 (Figura 3b) amplifica o bias úmido no setor central dos estados do Maranhão e Piauí (valores acima de $6 \mathrm{~mm} / \mathrm{dia}$ ), manteve a configuração semelhante no estado da Bahia, e estendeu as áreas de bias seco no Ceará (valores de 0 a $-2 \mathrm{~mm} / \mathrm{dia}$ ). O RAMS e o método análogo apresentaram um predomínio de bias seco ao longo do NEB, com valores da ordem de 0 a -2 mm/dia (Figuras $3 \mathrm{c}, 3 \mathrm{~d}$ ).

Para o trimestre MAM (Figura 4) as diferenças não foram muito marcantes. O ECHAM4.5 e o MRE/97 mantiveram as mesmas configurações de erro apresentadas no trimestre anterior, com predomínio de um bias úmido em grande parte do NEB (Figuras 4a, 4b).

Para o RAMS e o modelo análogo as configurações do bias e seus valores mostrados na Figura 4 também não apresentaram significativas mudanças. Apenas uma diferença mais marcante foi observada no resultado do método análogo nos setores centro-leste dos estados da Paraíba e Pernambuco, que apresentaram bias negativo no trimestre FMA e um bias positivo no trimestre MAM (Figuras 3d e 4d).

A magnitude dos bias nos dois trimestres é similar aos resultados de modelagem dinâmica dos estudos de Nobre et al. (2001), Roads et al. (2003), Druyan et al. (2002) e Seth e Rojas (2003), Alves et al. (2005) que analisaram resultados para a América do Sul.

As figuras 5 e 6 mostram o EMA do ECHAM4.5, do $M R E / 97$, para o RAMS e para o método análogo para os trimestres FMA e MAM. Em todas as figuras, tanto para FMA como para o MAM, o EMA oscila entre 0 e $4 \mathrm{~mm} /$ dia em grande parte do NEB. Exceto para o MRE/97, nas áreas de amplificação do bias setor central dos estados do Maranhão e Piauí e região 
noroeste do NEB cujos EMAs foram superiores a $4 \mathrm{~mm} /$ dia (Figuras $5 \mathrm{~b}$ e $6 \mathrm{c}$ ).

EMAs com valores superiores a $4 \mathrm{~mm} /$ dia foram mais abrangentes em áreas sobre o NEB, para os resultados do $R A M S$ e do método análogo para os trimestres FMA e MAM (Figuras 5c, 6c e 5d e 6d). Essas áreas se destacaram em ambos os semestres para o RAMS, nos setores oeste e adjacências dos estados do Maranhão e Piauí em direção a Amazônia e parte da região Centro-Oeste.

Para o método análogo, as áreas com EMAs maiores em magnitude do que $4 \mathrm{~mm} /$ dia abrangem todo o Maranhão e parte dos estados do Tocantins e Pará, e algumas pequenas áreas no estado do Ceará e oeste do estado da Paraíba (Figuras 5d e 6d). Nessas figuras também se ressalta pequenas áreas isoladas com EMA com valores superiores a $10 \mathrm{~mm} /$ dia nos trimestres FMA e MAM nos estados do Ceará, Rio Grande do Norte e Bahia.

A Figura 7 mostra o mapa síntese para os trimestres FMA e MAM. Observa-se que para os dois trimestres há uma manutenção das áreas com menor EMA dos modelos apresentados e do método análogo. Para o ECHAM4.5, predomina áreas nos estados do Maranhão, Tocantins, Pará e parte de Goiás em FMA, e uma área sobre o estado da Bahia para MAM. Menores
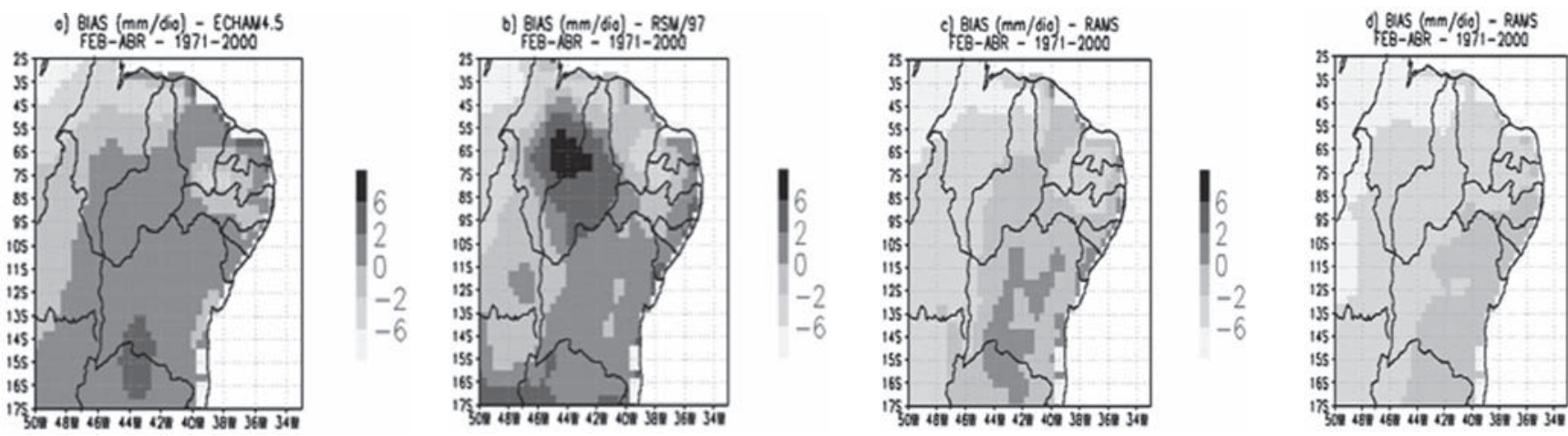

Figura 3 - bias (mm) para FMA (1971-2000). (a) ECHAM4.5, (b) MRE/97, (c) RAMS e (d) análogo.
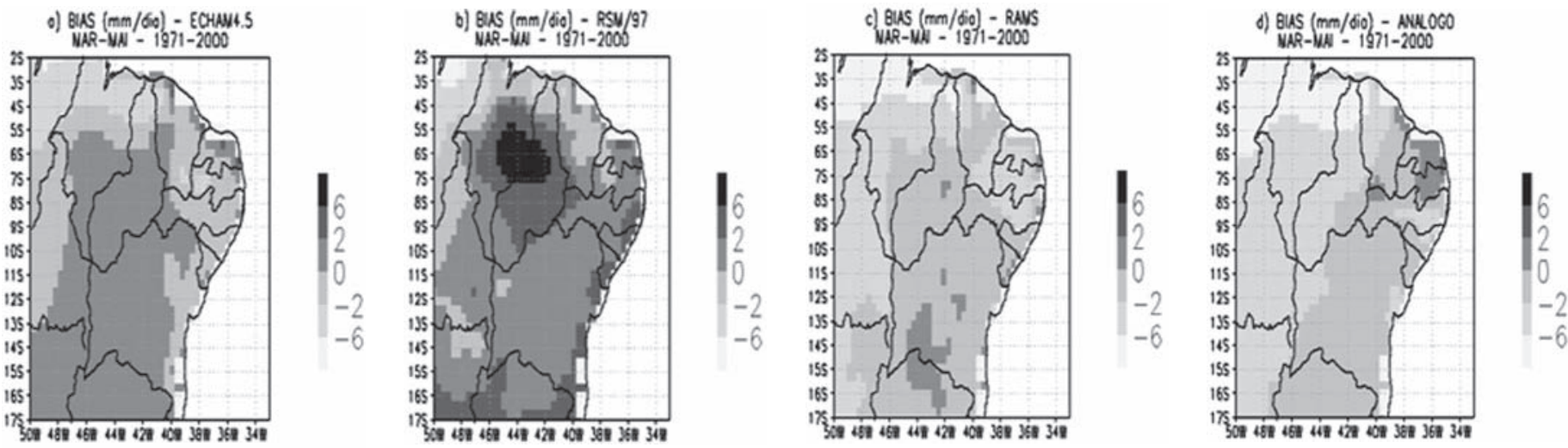

Figura 4 - bias (mm) para MAM (1971-2000). (a) ECHAM4.5, (b) MRE/97, (c) RAMS e (d) análogo.
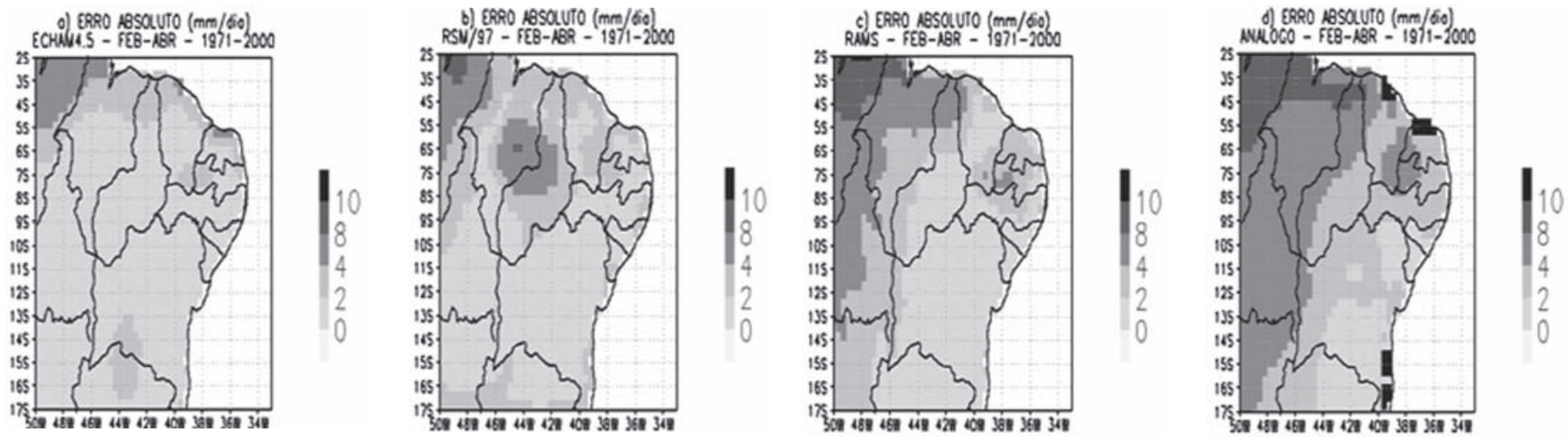

Figura 5 - EMA (mm) para FMA (1971-2000). (a) ECHAM4.5, (b) MRE/97, (c) RAMS e (d) análogo. 
EMAs para o MRE/97, centro-oeste do Rio Grande do Norte, Paraíba e Pernambuco e áreas isoladas da Bahia, Pará, Goiás, Tocantins e em Sergipe e Alagoas.

Para o método análogo, menores EMAs foram observados no norte do Maranhão e Piaú e oeste e sul em áreas do Ceará, centro-sul do Piauí, oeste e nordeste da Bahia. Os resultados do RAMS foram os que apresentaram menores áreas ao longo do NEB e vizinhanças com menores EMAs nos trimestres FMA e MAM, destacaram-se áreas no estado da Bahia, Ceará e áreas isoladas no litoral leste do NEB.

Embora não tenha sido objeto de análise mais específica nesse estudo, aqui é feita uma inferência sobre a parametrização de convecção que é usada nos modelos dinâmicos. Essa parametrização, ou sua resolução numérica nesses modelos é um dos fatores principais no resultado final da precipitação obtida pela modelagem, em particular, na região tropical onde grande parte da precipitação deve-se a formação de nuvens convectivas (Costa et al., 1998). É provável que a variação do bias dos modelos numéricos apresentados acima seja devido a essa questão.

O artigo de Arakawa (2004) mostra que desde o início da década de 1960 houve significativa evolução na parametrização e resolução desses processos de convecção nos modelos numéricos. Mas mesmo assim os diferentes métodos usados atualmente ainda não conseguem resolver, e se parametrizados (valores vindos de simulações ou observações), terem uma boa aproximação de todos os processos físicos que envolvem a interação das nuvens com o meio ambiente. Arakawa (2004) aponta alguns processos físicos que são cruciais para estudos de parametrização e resolução da interação do que ocorre na escala de nuvens e a atmosfera adjacente. Estes poderão melhorar a resposta física dos modelos dinâmicos entre os quais podem ser citados: distribuição vertical do aquecimento e resfriamento por nuvens cumullus, transporte de massa por convecção cumullus, interações da camada de nuvens com a camada limite planetária e com os processos radiativos, interações mecânicas com fluxo
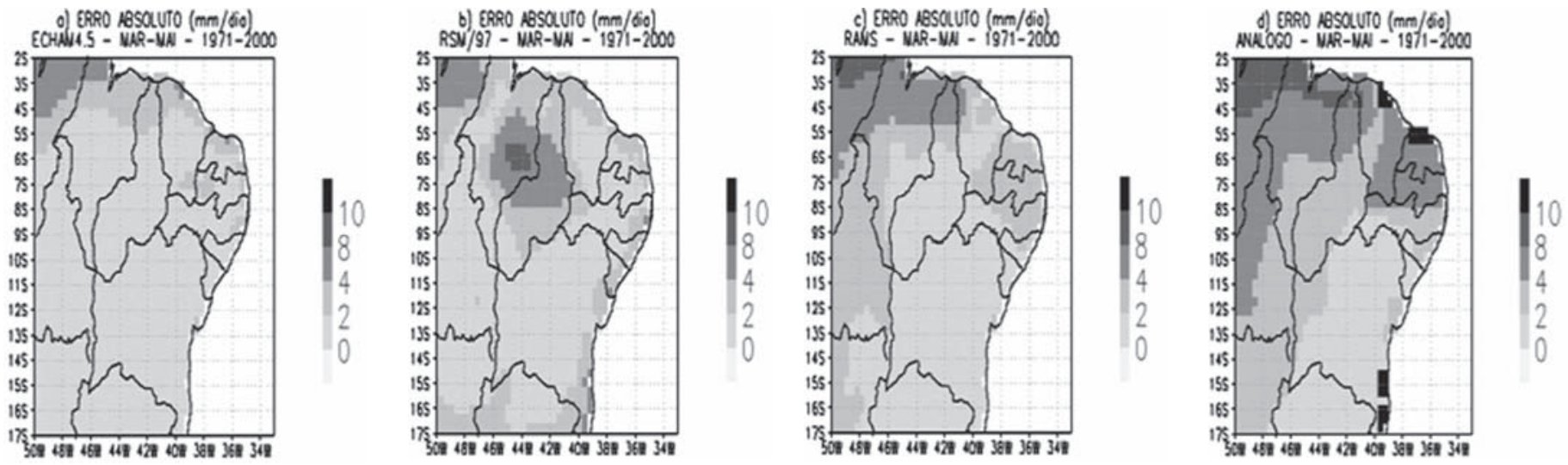

Figura 6 - EMA para MAM (1971-2000). (a) ECHAM4.5, (b) MRE/97, (c) RAMS e (d) análogo.
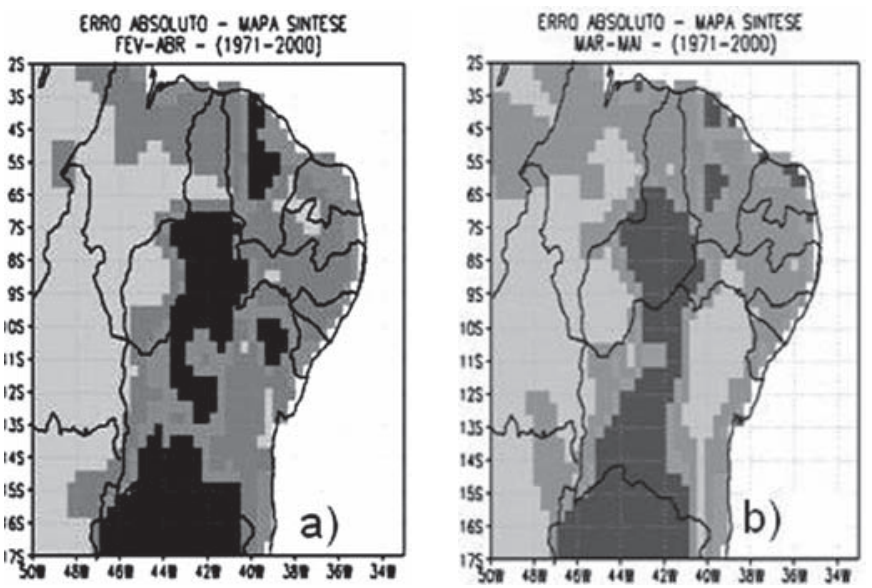

Figura 7 - Mapa síntese em função do menor EMA (mm/dia) em magnitude para os trimestres. (a) FMA e (b) MAM. O gradiente em tons de cinza mais claro ao cinza mais escuro indicam áreas de menor EMA para o ECHAM4.5 para o MRE/97 para o método análogo e para o RAMS. 
médio e a inclusão de efeitos não determinísticos (estocásticos). A Figura 2 de Arakawa (2004) mostra uma das principais questões relacionadas às incertezas nas formulações de nuvens e seus processos físicos associados, ainda objetos de estudo, que deverão ser inseridas nos modelos dinâmicos para melhorar a resolução da convecção tropical.

Muitos estudos e experimentos de microfisica de nuvens nessa questão são necessários, por exemplo, esperar-se-iam que os erros do modelo RAMS fossem menores em magnitudes do que o ECHAM4.5 e o MRE/97, porquê o RAMS foi simulado com uma microfisica de nuvens completa, fato não observado nos resultados exceto em algumas áreas do NEB.

A Figura 8 mostra a média, o bias e o desvio padrão em mm/dia para os períodos FMA e MAM dos resultados dos modelos ECHAM4.5, MRE/9, RAMS e para o método análogo para um área representativa do setor norte do NEB $\left(2^{\circ} \mathrm{S}-12^{\circ} \mathrm{S}\right.$ e $\left.45^{\circ} \mathrm{W}-37^{\circ} \mathrm{W}\right)$.

Para ambos os trimestres FMA e MAM o ECHAM4.5 e o $M R E / 97$ apesar de superestimarem a média no SNNEB, apresentaram valores mais próximos do observado com um bias úmido em torno de $1 \mathrm{~mm} /$ dia, enquanto o RAMS e o método análogo subestimaram a média observada e apresentaram um bias seco, maior em magnitude na estimativa do método análogo (em torno de -2 mm/dia). A estimativa do ECHAM4.5 apresentou um bias mais próximo do observado, enquanto o $R A M S$ e método análogo apresentaram o menor e o maior desvio padrão para ambos os trimestres.

a) FEV-ABR (45W-35W \& 2S-12S) - 1971-2000

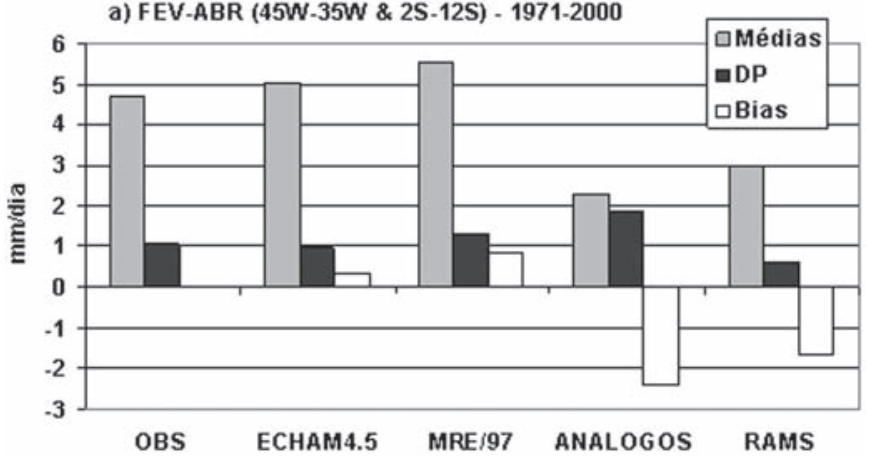

b) MAR-MAI (45W-35W \& 2S-12S) - 1971-2000

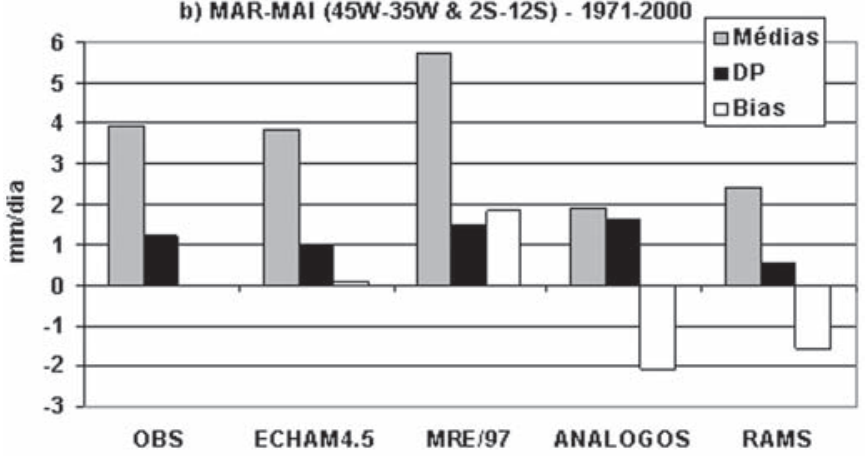

Figura 8 - Média, bias e desvios padrão(DP), para uma área represen- tativa do setor norte do NEB $\left(2^{\circ} \mathrm{S}-12^{\circ} \mathrm{S}\right.$ e $\left.45^{\circ} \mathrm{W}-37^{\circ} \mathrm{W}\right)$. a) para fevereiro a abril (FEV-ABR); b) março a maio (MAR-MAI).

Os bias tanto dos modelos numéricos como do método análogo foram menores do que os apresentados em Moura e Hantenrath (2004), quando comparados aos resultados do modelo dinâmico ECHAM4.5 (ver Tabela 3 de Moura e Hastenrath, 2004). Nesse estudo, esses autores mostraram uma comparação entre os resultados da modelagem dinâmica (ECHAM4.5), e os resultados de modelagem empírica, sistema desenvolvido na Universidade de Maryland. Esse sistema usa como preditores para precipitação no NEB, variáveis como anomalias de TSM no Pacífico e Atlântico Tropical, componente meridional do vento no Atlântico Tropical entre os meses de outubro a janeiro. Detalhes deste sistema são encontrados em (Greischar, 1993b; Greischar e Hastenrath, 2000). A diferença é que o período de análise no estudo de Moura e Hastenrath (2004) foi de um quadrimestre (março a junho).

Nas figuras 9, 1011 e 12 apresenta-se o Heidke Skill dos modelos ECHAM4.5, MRE/97, RAMS e do método análogo para as três categorias definidas individualmente e para as três categorias em conjunto como $\mathrm{S}, \mathrm{N}$ e C para o período fevereiro a maio. Para a categoria $\mathrm{S}$, observa-se que o ECHAM4.5 e o MRE/97 apresentam maiores skill acima de 0,4 no centro-norte do NEB, incluindo a região semi-árida, com alguns valores superando 0,6 nos estados do Ceará, Piauí, Maranhão, Rio Grande do Norte e Alagoas. Para o RAMS os valores de Skill maiores que 0,4 diminuíram em área, localizando-se, nas áreas próximas ao litoral norte do NEB. Na área centro-norte da Bahia o RAMS apresentou melhor Skill do que os outros modelos. O método análogo tem baixo Skill entre 0,1 e 0,2, e com configuração homogênea em todo o NEB. Áreas com Skill aleatório (valores menor do que zero) foram mais freqüentes no ECHAM4.5 e RAMS na direção da Região Centro-Oeste do país.

Na Figura 10 que mostra $o$ Heidke Skill para a categoria $\mathrm{N}$ pode-se observar para todos o modelos (ECHAM4.5, MRE/97 e RAMS) uma mesma ordem de magnitude dos valores de Skill em todo o NEB. Além disso, houve um aumento nesses modelos com áreas de Skill menor que zero. Para o caso do método análogo, embora tenha se mantido a homogeneidade dos valores ao longo da NEB, houve um aumento em magnitude com os valores oscilando entre 0,2 e 0,3, com algumas áreas na Bahia, leste da região e litoral norte do Ceará e Maranhão e sul do Piauí com valores superiores a 0,3 .

Para a categoria C (Figura 11), as características em magnitudes do Heidke Skill foram semelhantes às observadas para a categoria seco para o ECHAM4.5 e MRE/97, exceto o aumento em área de Skill com valores acima de 0,4 sobre o estado do Ceará para o ECHAM4.5 e valores acima de 0,7 no setor central dos estados do Ceará e Piauí. Essa área no Piauí coincide com área de elevado bias úmido mostrado na Figura 4b. O modelo 
RAMS apresentou maiores Skill em áreas do Ceará e Rio Grande do Norte com valores entre 0,5 e 0,6 com pequenas áreas excedendo acima de 0,7 . O modelo análogo teve o mais baixo Skill em todas as outras categorias, com valores homogêneos em todo o NEB oscilando em grande parte da região entre 0 e 0,1 mesmo assim um pouco mais alto que o Skill aleatório.

Para as três categorias conjuntas (Figura 12), os modelos com melhores Skill foram o ECHAM4.5 e MRE/97, persistindo nas áreas do setor norte do NEB valores acima de 0,3 e 0,4, e algumas áreas dos estados do Ceará e Piauí com valores acima de 0,5. Para o RAMS Heidke Skill com valores acima 0,4 foram observados em áreas isoladas no norte do NEB. No método análogo como observado nas categorias $\mathrm{S}, \mathrm{N}$ e $\mathrm{C}$ os valores do Heidke Skill foram baixos entre 0 e 0,1 , mantendo as características homogêneas ao longo do todo o NEB.

Os menores valores de Heidke Skill para a categoria N em todo o NEB são consistentes com resultados de modelagem dinâmica para o NEB (Sun et al., 2005; e outros). Essa característica deve-se a forçante de contorno dos modelos (TSM), como nos anos considerados $\mathrm{N}$ não há nenhuma condição tér-
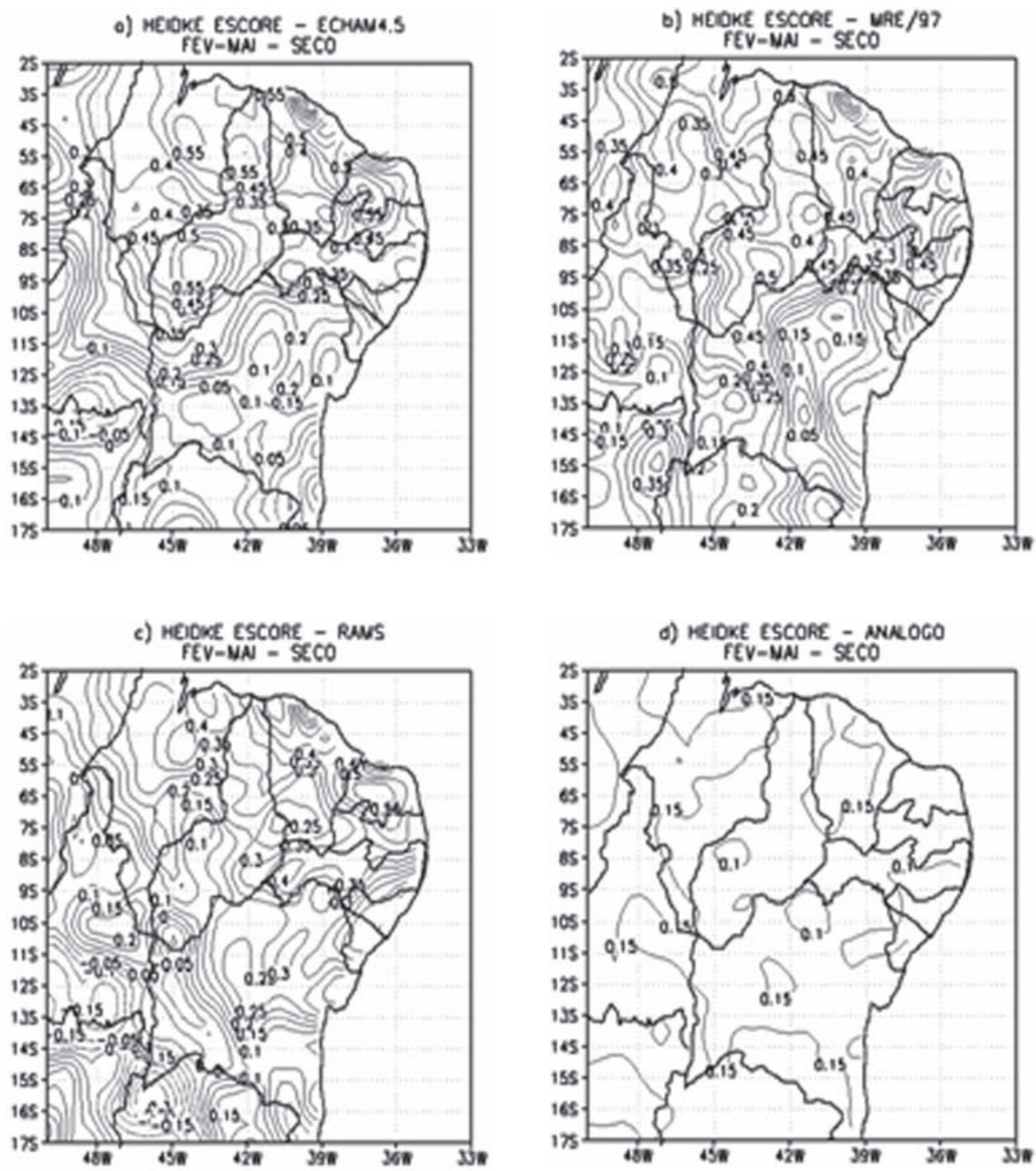

Figura 9 - Heidke Skill para o para categoria Seco no período FEV-MAI. (a) ECHAM4.5; (b) MRE/97; (c) RAMS e (d) análogo. 
mica dominante, por exemplo, La Niña (El Niño) que poderiam forçar a atmosfera a uma resposta como de mais chuva (menos chuva) no NEB (Moura \& Shukla, 1981; Nobre e Shukla, 1986); a resposta na distribuição de chuva nesses anos $\mathrm{N}$ não tende a se direcionar para nenhum extremo, sendo mais aleatória. Esse fato explica também por que para nas categorias $\mathrm{S}$ e $\mathrm{C}$ os Heidke Skill são maiores, principalmente, na região semi-árida do NEB cujas chuvas são controladas em grande parte pelas condições térmicas do Oceano Pacífico e Atlântico Tropical. Isto é, quando a ocorrência dessas categorias, em geral, estas se associam aos eventos de El Nino (La Niñas) e a distribuição de chuvas no NEB tende a direcionar-se a um dos extremos seco (chuvoso).

Uma causa do baixo Heidke Skill do método análogo e sua característica homogênea de magnitude de valores ao longo do NEB sugere o tempo não ideal de identificação dos
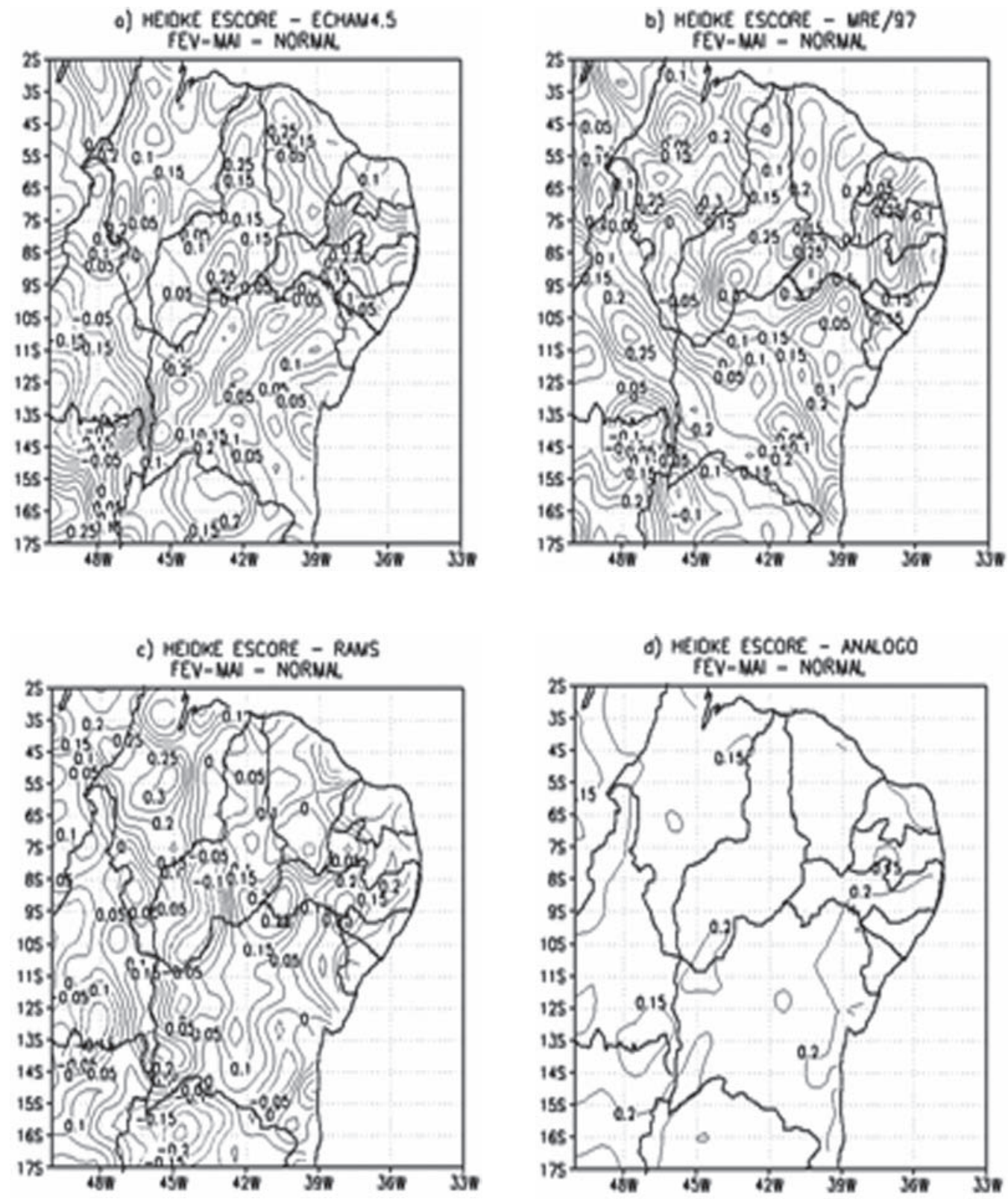

Figura 10 - Heidke Skill para o para categoria Normal no período FEV-MAI. (a) ECHAM4.5; (b) MRE/97; (c) $R A M S$ e (d) análogo. 
análogos, com dois meses de antecedência (novembro) para o início da quadra chuvosa do setor norte do NEB (fevereiro a maio). Como grande parte da precipitação nessa região, principalmente no período MAM é explicado pelas condições térmicas no Atlântico Tropical simultâneas (observadas no mesmo período de MAM) da ordem de $64 \%$ da variabilidade (Moura e Shukla, 1981; Nobre e Shukla, 1996, entre outros), com certeza esse período para a identificação dos análogos não é o ideal. Fato que não ocorre nos modelos dinâmicos, pois a TSM usada foi à observada nos respectivos meses, como mencionado na metodologia a chamada condição de contorno da "previsão ótima". A essa questão, somam-se também indícios que os padrões de anomalias de TSM, que em geral, estão associados à definição da qualidade da estação chuvosa do setor norte do NEB se desenvolvem melhor a partir de fevereiro (Curtis e Hastenrath, 1985).
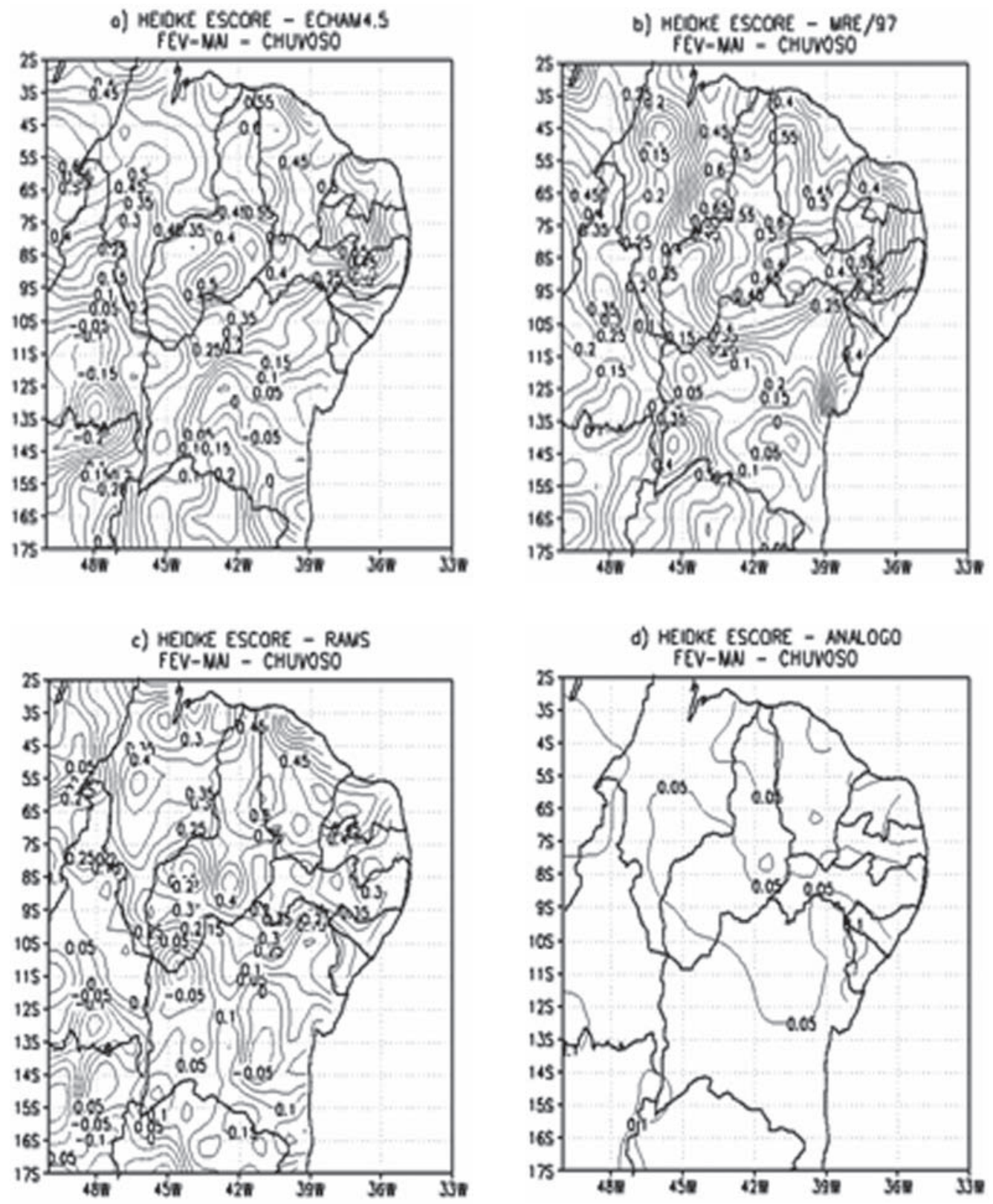

Figura 11 - Heidke Skil para a categoria Chuvosa no período FEV-MAI. (a) ECHAM4.5; (b) MRE/97; (c) $R A M S$ e (d) análogo. 

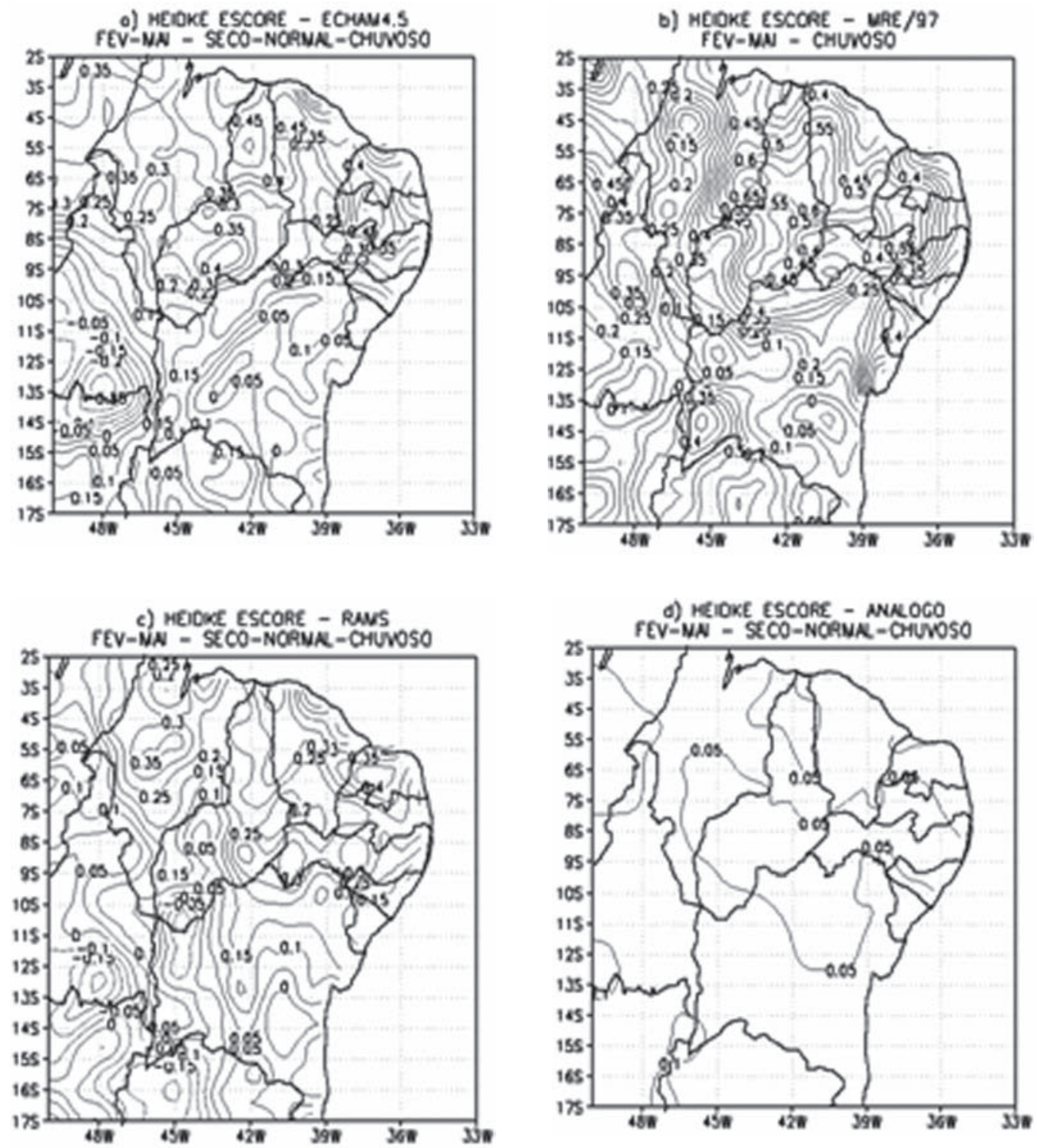

Figura 12 - Heidke Skill para as três categorias Seco, Normal e Chuvoso no período FEV-MAI. (a) ECHAM4.5; (b) MRE/97; (c) RAMS e (d) análogo.

\section{CONCLUSÕES E RECOMENDAÇÕES}

Embora, os resultados dos modelos dinâmicos tenham sido forçados com as TSMs observadas (diagnóstico ou a chamada "previsão perfeita"), e os resultados do método análogo foi uma previsão (usou-se os dados de novembro do ano anterior das anomalias de TSM nas áreas Niño 3 e setores norte e sul do dipolo no Atlântico Tropical) algumas conclusões importantes podem ser retiradas do estudo.

O EMA, o ECHAM4.5 teve menores erros em áreas dos estados do Maranhão, Tocantins, Pará e parte de Goiás em FMA, e uma área sobre o estado da Bahia para MAM. O
MRE/97 apresentou menores EMAs no centro-oeste do Rio Grande do Norte, Paraíba e Pernambuco e áreas isoladas da Bahia, Pará, Goiás, Tocantins e em Sergipe e Alagoas em ambos os trimestres. Para o método análogo, menores EMAs foram observados no norte do Maranhão e Piauí, oeste e sul do Ceará, centro-sul do Piauí e setores oeste e nordeste da Bahia, enquanto o RAMS mostrou menores áreas ao longo do NEB e vizinhanças com menores EMAs nos trimestres FMA e MAM, destacaram-se áreas no estado da Bahia, Ceará e áreas isoladas no litoral leste do NEB.

Quanto ao bias, o ECHAM4.5 e o MRE/97 mostraram um bias úmido em grande parte do NEB, porém com um valor 
médio para uma setor norte do NEB $\left(2^{\circ} \mathrm{S}-12^{\circ} \mathrm{S}\right.$ e $\left.45^{\circ} \mathrm{W}-37^{\circ} \mathrm{W}\right)$ mais próximo do observado. Para o RAMS e o método análogo houve um predomínio de um bias seco sobre o NEB, com valores em magnitudes mais distantes do observado para a área citada acima.

A previsibilidade (Heidke Skill) em três categorias S, N e $\mathrm{C}$ para o período de fevereiro a maio mostrou que os modelos dinâmicos ECHAM4.5 e os regionais MRE/97 e RAMS superaram em valores, da ordem de 0,4 a 0,5 , o método análogo que apresentou características homogêneas em todo o NEB com valores em torno de 0,1 a 0,2 . O modelo RAMS apresentou um maior Heidke Skill em algumas áreas do centro-norte do Estado da Bahia do que os modelos ECHAM4.5 e MRE/97 para a categoria S. Os maiores Heidke Skill foram observados para os setores central do Estado Piauí, oeste do Estado do Ceará e áreas isoladas do Rio Grande do Norte e Pernambuco para a categoria C para o MRE/97. Áreas isoladas também com valores acima de 0,6 foram observadas para categoria $\mathrm{S}$ nos estados do Maranhão, Piauí, Ceará, Rio Grande do Norte e Alagoas.

Para estudos futuros sugerem-se novos testes com defasagens mensais, trimestrais até quadrimestrais para identificação dos análogos, o uso de mais análogos (15, 20 ou mais). Além disso, podem ser testados novos métodos estatístico-estocásticos como, por exemplo, os auto-regressivos que usam como variáveis preditoras não só as condições termodinâmicas nos Oceano Pacífico e Atlântico Tropical, além de mais variáveis atmosféricas (convergência de umidade, ventos junto à superfície, vorticidade, etc.) sobre a o setor norte do NEB. Para a modelagem dinâmica sugere-se o acoplamento de multi-modelos (MCGAs e outros modelos regionais), além de testes com TSMs persistidas e previstas bem com o aumento dos membros da simulação.

\section{AGRADECIMENTOS}

Os autores agradecem aos Editores e Revisores da RBMET pelas críticas e sugestões que melhoraram a apresentação e o conteúdo científico do artigo.

\section{REFERÊNCIAS BIBLIOGRÁFICAS}

ALVES, J. M. B.; CAMPOS, J. N. B.; FILHO, F. A. S.; MONCUNNIL, D. F.; SILVA, E. M.; BARBOSA, W. L.; FERREIRA, A. G.; SUN, L.; MOURA, A. D. Uma avaliação preliminar entre simulações climáticas de um modelo global (ECHAM4.5) e um donwscaling dinâmico para o setor norte da Região Nordeste do Brasil. Rev. Bras. Meteor.., v.20, n.2, p.40-55, 2005.
ALVES, J. M.B.; BRISTOT, G.; COSTA, A. A.; MONCUNNIL, D. F.; NÓBREGA, D. S. B.; SANTOS, A. C. S.; SILVA, E. M.; MELCÍADES, W. L. B.; FILHO, V. P. S.; SOUZA, I. A. Uma aplicação da técnica de downscaling dinâmico no setor norte da Região Nordeste do Brasil. Rev. Bras. Meteor., v.18, n.2, p.161-180, 2003.

ALEXANDER, G. D., AND COTTON, W. R. The use of cloud resolving simulations of mesoscale convective systems to build a mesoscale parameterization scheme. J. Atmos. Sci., 55, 2137-2161, 1998.

ANDERSON, J. H; Van den Dool; BARNSTON, A.; CHEN, W.; STERN, W.; PLOSHAY, J. Present -day capabilities of numerical and statistical models for atmospheric extratropical seasonal simulation and prediction. Bull. Amer. Meteor. Socie., v.80., p.1349-1361. 1999.

ARAKAWA, A. The cumulus parameterization problem: Past, present and future. J. Climate, v.17, n.13, p. 2493-2525. 2004.

BAMZAI, A. S.; SHUKLA, J. Relation between Eurasian snow cover, snow depth, and the Indian summer monsoon: An observational study. J. Climate, v.12, p.3117-3132. 1999.

BARNSTON, A.; DOLL, H. M. v. d.; RODENHUIS, D. R.; ROPELEWSKI, C. F.; KOUSKY, V. E.; O’LENIC, E. A.; LIVEZEY, R. E.; ZEBIAK, S. E.; CANE, M. A BARNETT, T. B.; GRAHAM, N. E.; JI, M.; LEETMAA, A. Long-lead seasonal seasonal forecasts-Where do we stand? Bull. Amer. Meteor. Socie., v.75., n.11, p.2097-2114. 1994.

BARNSTON, A.; GLANTZ, M. H.; HE, Y. Predictive skill of statistical and dynamical climate models in SST forecasts during the 1997-98 El Niño episode and the a998 La Niña onset. Bull. Amer. Meteor. Socie., v.20, p.217-243. 1999.

BUISHAND, T. A.; BRANDSMA, T. Multi-site simulation of daily precipitaion and temperature in the Rhine basin by nearest-neighbor resampling. Water Resour. Res. v.37, p.2761-2776. 2001.

CARDOSO, G. B. B. 2003. Uso de otimização/simulação e previsão de afluências na operação tática dos reservatórios do sistema Jaguaribe-Metropolitano. Dissertação de Mestrado. Universidade Federal do Ceará. Departamento de Engenharia Hidráulica e Ambiental. 141p. 
CARSON, D. J. Seasonal forecasting. Quart. Jour. Roy. Meteor. Socie. v.124, p.1-26. 1998.

CAVALCANTI, I. F. A.; MARENGO, J. A.; SATYMURTY, P.; NOBRE, C. A.; TROSNIKOV, I.; BONATTI, J. P.; MANZI, A. O.; TARASOVA, T.; PEZZI, L. P.; D'ALMEIDA, C.; SAMPAIO, G.; CASTRO, C. C.; SANCHES; M. B.; CAMARGO, H. Global climatological features in a simulation using CPTEC/COLA AGCM. J. Climate, v.15, n. 21, p.2965-2988. 2002.

CHEN, C.; COTTON, W. R. A. One-dimensional simulation of the stratocumulus coupled mixed layer. Boun. Layer Meteor., v.25, p.289-321. 1983.

CHEN, C. AND COTTON, W.R.; The physics of the marine stratocumulus capped mixed layer, J. Atmos. Sci., v.44, p.2951-2977. 1987.

CHOU, S. C.; NUNES, A. M. B.; CAVALCANTI, I. F. A. Extend range forecast over South America using the regional eta model. J. Geophys. Res., v.105, d8, p.10.147-10160. 2000.

CLAUSSEN, M. U. et al. A global data set of land-surface parameters. Max Planck Institut für Meteorologie, Report n.135, Hamburg, Germany, 23pp. 1994.

COSTA, A. A. Relatório de atividades do Projeto MONUMENT (Modelagem Numérica de Mesoescala Aplicada à Previsão Climática no Nordeste Brasileiro). Fundação Cearense de Meteorologia e Recursos Hídricos. Fortaleza-Ce. 24p. 2006.

COSTA, A. A., COTTON, W. R., WALKO, R. L., PIELKE, R. A., Coupled ocean-cloud-resolving simulations of the air-sea interaction over the western Pacific. J. Atmos. Scie., v. 58, p. 3357-3375, 2001a.

COSTA, A. A., COTTON, W. R., WALKO, R. L., PIELKE, R. A., JIANG, H., SST sensitivities in multi-day TOGA COARE simulations. J. Atmos. Scie., v.58, p. 253-268, 2001 b.

CURTIS, S.; HASTENRAH, S. Forcing of anomalous sea surface temperature evolution in the tropical Atlantic during Pacific warm events. J. Geophy. Res., v.100,(C*), p.15835$15847,1995$.

DRUYAN, L. M.; FULAKEZA, M.; LONERGAN, P. Dynamic downscaling of seasonal climate predictions over Brazil. J. Climate, v.15, n.23, p.3411-3426. 2002.
FOLlAND, C. W. K.; COLMAN, A. W.; ROWELL, D. P; DAVEY, M. K. Predictability of northeast Brazil rainfall and real-time forecast skill. J. Climate, v.14, p.1937-1978. 2001.

GIORGI, F.; BATES, G. T. On climatological skill of a regional model over complex terrain. Mon. Wea. Rev., v.117, p.23252347. 1989.

GIORGI, F. On simulation of regional climate using a limited area model nested in a general circulation model. J. Climate, v.3, p.941-963. 1990.

GODDARD, L.; ZEBIAK, S. E.; ROPELEWSKI, C. F.; BASHER, R.; CANE, M.A. Current approaches to seasonalto-interannual climate predicitions. Inter. Jour. Climatolo., v.21, p.1111-1152. 2001.

GREISCHAR, L.; HASTENRATH, S. The rainy seasons of the 1990s in northeast Brazil: Real-time forecasts and verification. J. Climate, v.13, p.3821-3826. 2000 .

HANSEN, J. W.; HODGES, A. W.; JONES, J. W. ENSO influence on agriculture in the Southeastern United States. J. Climate. v.11, n.3, p.404-411. 1998.

HASTENRATH, S.; HELLER, L. Dynamics of climatic hazards in northeast Brazil Quart. Jour. Roy. Meteor. Socie., v.103: 77-92, 1977.

HASTENRATH, S. On climate predicition in the tropics. Bull. Amer. Meteor. Soc., v.67, p.692-702. 1986.

HASTENRATH, S. Climate and Circulation of the Tropics. Reidel, 455p. 1995.

HASTENRATH, S. Recents advances in tropical climate prediction. J. Climate., v.8, p.1519-1532. 1995a.

HASTENRATH, S. Climate Dynamics of the Tropics. Kluwer., 488p. 1995 b.

HASTENRATH, S. Climate prediction (empirical and numerical). Encyclopedia of Atmospheric Sciences. J. Holton et al., Eds. Academic Press, 411-417. 2002.

HASTENRATH, S.; GREISCHAR, L. Further work on the predicition of northeast Brazil rainfall anomalies. J. Climate, v.6, p.743-758. 1993a. 
HASTENRATH, S.; GREISCHAR, L. Circulation mechanisms related to northeast Brazil rainfall anomalies. J. Geophy. Res., v.98, (d3), p. 5093-5102. 1993b.

HARRINGTON, J. Y. The effects of radiative and microphysical processes on simulated warm and transition season Artic stratus. Ph.D. dissertation, Atmps'heric science. Paper No. 637, Colorado Statte Univertsity, Dept. of Atmospheric Science, 289p. 1997.

HONG, S. -Y.; PAN, H. -L. Nonlocal boundary layer vertical diffusion in a medium-range forecast model. Mon. Wea. Rev., v.124, p.2322-2339. 1996.

IREDELL, M.; PURSER, J. A local diffusion to control advective numerical instability in the NMC global spectral model. Preprints, $11^{\text {th }}$ Conf. on Numerical Weather Prediction, Norfolk, VA, Amer. Meteor. Soc., 22-23. 1994.

JUANG, H.-M. H.; KANAMITSU, M. The NMC nested regional spectral model. Mon. Wea. Rev., v.122, n.1, p.326. 1994

KANAMITSU, M. Description of the NMC global data assimilation and forecast system. Wea. Forecast., v4. n.3, p.335-342. 1989.

KANAMITSU, M. et al. Recent changes implemented into the global forecast system at NMC. Wea. Forecast., v.6, n.1., p.435-455. 1991.

KANAMITSU, M, S-Y., HONG 1997. The NCEP regional spectral model: An update. Bull. Amer. Meteor. Socie. v.78, n.10, p.2125-2143. 1997.

KANAMITSU, M.; KUMAR, A.; JUANG, H. H.; SHEMM, J.; WANG, W.; YANG, F.; HONG, S.; PEING, P.; CHEN, W.; MOORTHI, S.; JI, M. NCEP dynamical seasonal forecast system 2000. Bull. Amer. Meteor. Socie., v.83, n.7, p.1019-1037. 2002.

KUO, H. L. Further studies of the parameterization of the influence of cumulus convection on large scale flow. J. Atmos. Sci., v.31, p.1232-40. 1974.

LALL, U.; SHARMA, A. A nearest neighbor bootstrap for resembling hydrologic time series. Water Resour. Res., v.32, p.679-693. 1996.
LATIF, M. et al. A review of the predictability and prediction of ENSO. J. Geophy. Res., v.103, p. 14375-14393. 1998.

LOUIS, J. -F.; J. TIEDTKE; -F. GELEYN. A short history of the PBL parameterization at ECMWF. Proceedings of the ECMWF Workshop on Planetary Boundary Layer Parameterization. November. European Centre for MediumRange Weather Forecasts, Reading, England, 59-80. 1981.

MARENGO, J.; CAVALCANTI, I, F. A.; SATYAMURTY, P. et al.Assessment of regional seasonal rainfall predictability using the CPTEC/COLA atmospheric GCM. Climate Dynamics, v.21, p.1877-1891. 2003.

MOURA, A.D.; SHUKLA, J. On the dynamics of droughts in northeast Brazil: Observations, theory and numerical experiments with a general circulation model. J. Atmos. Sci., v.38, n.7, p.2653-2675, 1981.

MOURA,A. D.; HASTENRATH, S. Climate prediction for Brazil's Nordeste: Performance of empirical and numerical modeling methods. J. Climate, v.17, n.13, p.2627-2672. 2004.

NAIR, U. S.; HJELMFELT, M. R.; PIELKE, Sr., R. A. Numerical simulations of the 9-10 june 1972 Black Hills storms using CSU-RAMS. Mon. Wea. Rev., n.125, p.17531776. 1997.

NEW, M. G.; HULME, M; JONES. P. D. Representing twentieth century space time climate variability. Part II: Development of a 1901-1996 monthly terrestrial climate fields. J. Climate, v.13, p.2217-2238. 2000.

NOBRE, P.; SHUKLA, J. Variations of sea surface temperature, wind stress and rainfall over the tropical Atlantic and South America. J. Climate, v.10, n.4): 2464-2479, 1996.

NOBRE, P.; A. D. MOURA.; L. SUN. Dynamical downscaling of seasonal climate prediction over Nordeste Brazil with ECHAM3 and NCEP'S Regional Spectral Model at IRI. Bull. Amer. Meteor. Socie., v.82, p.2787-2796. 2001.

OLSSON, P. Q.; COTTON, W. R. Balanced and unbalanced circulations in a primitive equation simulation of a midlatitude MCC. Parte I: The numerical simulation. J. Atmos. Sci., v.54, p.457-478. 1998.

OLSSON, P. Q. et al. Exploratory cloud-resolving simulations of boundary-layer Artic stratus clouds. Part I: Warm season clouds. Atmos. Res., v.47-48, p.573-597. 1998. 
PAN, H. -L.; L. MARTH. Interaction between soil hydrology and boundary layer development. Bound. Layer Meteor, (38), p.185-202. 1997.

PAN, H. -L.; W. -S. WU. Implementation a mass flux convective parameterization package for the NMC medium-range forecast model. Preprints, $10^{\text {th }}$ Conf. on Numerical Weather Prediction, Portland, OR, Amer. Meteor. Soc., p.96-98. 1994.

PALMER, T. N.; ANDERSON, D. L. T. The prospect for seasonal forecasting - A review paper. Quart. Jour. Roy. Meteor. Society, v.120, p.755-793. 1994.

PIELKE, R. A. et al. A comprehensive meteorological modeling system RAMS. Meteor. Atmos. Physi., p.69-91. 1992.

RAJAGOPALAN, B.; LALL, U. A K-nearest-neighbor simulation for daily precipitation and other variables. Water Resour. Res., v.35, p.3089-3101. 1999.

ROADS, J. et al. International Research Institute/Applied Research Centers (IRI/ARCs) regional model intercomparison over South America. J. Geophy. Res, v.108, p.4425, doi:10.1029/2002JD003201. 2003.

ROECKNER, E. The atmospheric general circulation model ECHAM4: model description and simulation of the presentday climate. Max Planck Institut für Meteorologie, Report n.218, Hamburg, Germany, 90. 1996.

SETH, A.; ROJAS, M. Simulation and sensitivity in a nested modeling systems for south América. Part I: Re-analyses boundary forcing. J. Climate, v.16, p. 2698-2712. 2003.

SILVA FILHO, V. P. Previsão de vazão no Semi-Árido Nordestino, utilizando modelos atmosféricos: Um estudo de Caso. Tese de Doutorado. Universidade Federal do Ceará. Departamento de Engenharia Hidráulica e Ambiental. Fortaleza-Ce. 105p. 2005.

SOUZA FILHO, F. A. \& LALL, U. Seasonal to interannual ensemble stream flow forecast for Ceará, Brazil: Applications of multivariate, semi parametric algorithm. Water Resour. Res., 39(11), p.1307, doi: 10.1029/2002WR001373. 2003.

SOUZA FILHO, F. A.; LALL, UPMANU. Modelo de Previsão de Vazões Sazonais e Interanuais. Revista Brasileira de Recursos Hídricos, ABRH, Porto Alegre, RS, v. 9, n. 2, p. 61-74, 2004.
SUN, L.; MONCUNNIL, D. F.; LI, H.; MOURA, A. D.; FILHO, F. D. D. S. Climate downscaling over Nordeste Brazil using NCEP RSM97. J. Climate, v.18, p.551-567. 2005 .

YOUNG, K. C. A multivariate chain model for simulating climatic parameters from daily data. J. Appl. Meteor., v.33, p.661-671. 1994.

TIEDTKE, M. A. comprehensive mass flux scheme for cumulus parameterization in large-scale models. Mon. Wea. Rev., v.117, p.1779-1800. 1989.

TREMBACK, C. J. Numerical simulation of a mesoescale convective complex: model development and numerical results. Dissertation (Ph.D.), Colorado State University, Colorado, USA. 1990.

UVO, C. B; REPELLI, C. A.; ZEBIAK, S. E.; KUSHNIR, Y. The relationship between tropical Pacific and Atlantic SST and northeast Brazil monthly precipitation. J. Climate, v.13, p.287-293. 1998.

WALKO, R.L.; COTTON, W.R.; MEYERS, M.P. AND HARRINGTON, J.Y. New RAMS microphysics parameterization. Part 1: The single moment scheme, Atmos. Res., 38, 29-62, 1995b

WALKO, R.L., BAND, L.E.; BARON, J.; KITTEL, T.G.F.; LAMMERS, R.; LEE, T.J.; PIELKE, R.A.; TAYLOR, SR., C.; TAGUE, C.; TREMBACK, C.J. AND VIDALE, P.L. Coupled atmosphere-biophysics-hydrology models for environmental modeling. J. Appl. Meteor., v. 39, p.931944, 2000.

WILKS, D. S. Statistical Methods in the Atmospheric Sciences. Academic Press. San Diego. 464p. 1995.

ZIEGLER, C. L; LEE, T. L.; PIELKE, R. A. Sr. Convection initiation at the dry line: a modeling study. Mon. Wea. Rev., v.125, p.1001-1026. 1997.

XAVIER, T. de Ma. B. S.; XAVIER, A. F. S. 1998. Quantis para séries pluviométricas do Estado do Ceará e caracterização de períodos secos ou excepcionalmente chuvosos: 1964-1998. Fundação Cearense de Meteorologia e Recursos Hídricos. Fortaleza. Ceará., p.33. 